Pecvnia, 3 (2006), pp. 95-140

\title{
Comparación empírica de técnicas estadísticas para tablas de tres entradas: La construcción en Castilla y León en el periodo 2002-2004
}

\author{
Ma Jesús Mures Quintana \\ María Eva Vallejo Pascual \\ Ana García Gallego
}

\begin{abstract}
El objetivo de este trabajo es comparar las técnicas estadísticas más conocidas para el tratamiento de tablas de tres entradas. Para ello, se toman los ratios económico-financieros de las mayores empresas constructoras de la Comunidad Autónoma de Castilla y León (España) en el periodo 2002-2004. Aunque existen diferencias teóricas, las técnicas consideradas no arrojan resultados significativamente diferentes. Por otro lado, el estudio también demuestra la utilidad de este tipo de técnicas cuando se trabaja con información relativa a diferentes años.
\end{abstract}

Palabras clave: Datos cuantitativos; Tablas de tres entradas; Empresas constructoras; Castilla y León.
The target of this paper is the comparison of the best known statistical techniques for the treatment of Three-mode tables. Accordingly, the economic-financial ratios of the main building companies in the Autonomous Region of Castille and Leon (Spain) for the period 2002-2004 are taken into account. Although there are theoretical differences, the considered techniques do not release substantial different results. On the other hand, the study also shows the usefulness of this kind of techniques when working with information related to different years.

Key words: Quantitative Data; Three-mode tables; Building Companies; Castille and Leon. 


\section{INTRODUCCIÓN}

Desde el punto de vista estadístico, una tabla de tres entradas (individuos, variables, ocasiones $[n \times p \times T]$ ) puede considerarse como una sucesión de $T$ tablas de dos entradas (individuos, variables $[n \times p]$ ). Sin embargo, los métodos estadísticos que analizan éstas no están indicados para el tratamiento de las de tres entradas, ya que no permiten la comparación de los resultados individuales de las $T$ tablas.

En la literatura estadística existe un gran número de técnicas (o métodos) para el tratamiento de tablas de tres entradas, lo que hace difícil una visión organizada de las mismas. Las diferencias, desde el punto de vista teórico, entre algunas de estas técnicas ya han sido estudiadas por algunos autores. Concretamente Kiers (1988) compara los métodos de la escuela francesa y los anglosajones, haciendo especial hincapié en las relaciones entre Statis (Structuration des TAbleaux a Trois Indices de la Statistique), y CONDECOMP/PARAFAC. Casin (2001), por su parte, compara el Análisis de Componentes Principales Generalizado con el Análisis de Componentes Principales y con el Análisis Factorial Múltiple (AFM). Bove y Di Ciaccio (1994) realizaron un trabajo en el que se exponen las relaciones entre el método Statis y el modelo de Tucker2 (TUCKALS2). Por su parte, Pagès (1996) deduce los elementos de comparación entre el Análisis Factorial Múltiple y el método Statis.

Es evidente que la consideración de todos los métodos existentes sobrepasaría los límites de este trabajo, de aquí que tengamos que restringir nuestro estudio. Así, atendiendo a criterios de difusión y disponibilidad de software para su aplicación, hemos seleccionado para su comparación empírica algunos de ellos. Los métodos elegidos resultan apropiados para las tablas de tres entradas denominadas three-way data array (en las que los mismos $n$ individuos se observan sobre las mismas $p$ variables en las $T$ ocasiones). El objetivo es comprobar si las diferencias teóricas entre las técnicas se traducen o no en conclusiones empíricas diferentes.

Para lograr el objetivo planteado, en primer lugar se revisan brevemente, desde el punto de vista teórico, las técnicas o métodos que se van a considerar. A continuación, dichas técnicas se emplean para analizar la información económico-financiera relativa a las mayores empresas constructoras, según su cifra neta de negocio, en la Comunidad Autónoma de Castilla y León (España) en el periodo 2002-2004. El trabajo finaliza con las conclusiones más relevantes y las referencias bibliográficas. 


\section{METODOLOGÍA}

Los datos que constituyen las tablas de tres entradas se clasifican según tres índices, denominados modos o entradas y se expresan mediante la notación $x_{\mathrm{ijt}}$ :

$i=1 \ldots n$, expresa los individuos, objetos o unidades

$j=1 \ldots p$, expresa las variables o características

$t=1 \ldots T$, indica las situaciones, ocasiones o momentos de tiempo.

La tabla global resultante $X=\left\{x_{\mathrm{ijt}}\right\}$ (figura 1) puede considerarse como una sucesión de tablas de doble entrada $X^{t}$, cada una de las cuales referida a un momento (situación u ocasión) diferente. Por ello, se puede expresar como la sucesión de $X^{1}, X^{2} \ldots X^{\mathrm{T}}$, donde cada $X^{t}$ viene representada por el elemento $x_{\mathrm{ij}}$. Cuando cada $X^{t}$ está formada por los mismos $n$ individuos y las mismas $p$ variables, la tabla global de tres entradas se denomina three-way data array (según la terminología anglosajona). En la mayoría de las ocasiones las variables se centran y estandarizan, y cada matriz $X^{t}$ se transforma en $Z^{t}$, por lo que la tabla global resultante se denomina $Z=\left\{z_{\mathrm{ijt}}\right\}$.

Figura 1: Tabla de tres entradas: yuxtaposición de tablas de doble entrada

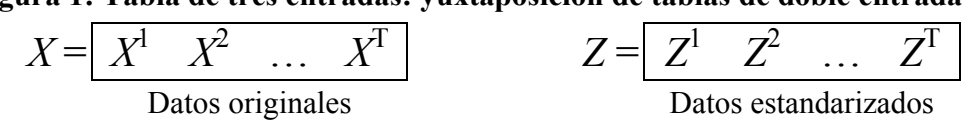

Desde una perspectiva descriptiva-exploratoria, se han desarrollado en las últimas décadas muchos métodos para analizar este tipo de tablas, algunos descritos en la literatura anglosajona y otros en la francesa. Sin embargo, muchos de los métodos desarrollados por la escuela francesa han sido ignorados por la literatura anglosajona, y a la inversa. En este caso, vamos a considerar una serie de ellos, sin tener en cuenta la escuela de procedencia, que son los más difundidos para analizar la información de naturaleza cuantitativa.

Los métodos que vamos a considerar pueden dividirse en asimétricos y simétricos. Los primeros se denominan así porque uno de los modos (generalmente las ocasiones) tiene un tratamiento diferente a los otros dos (individuos y variables), circunstancia que no se da en los métodos simétricos ya que tratan por igual los tres modos. Además, los asimétricos están dentro del denominado enfoque Interestructura-CompromisoIntraestructura $(\mathrm{ICl})$, nombre que se corresponde con las tres etapas en las 
que se desarrollan los dos métodos que vamos a considerar: el Statis (versión Statis y Statis dual) y el Análisis Factorial Múltiple (AFM). Los métodos simétricos se identifican con los denominados Métodos de componentes, siendo los más generales en la jerarquía ${ }^{1}$ el Tuker3 (TUCKALS3), Tuker2 (TUCKALS2) y PARAFAC/CONDECOMP. También se considera como método simétrico el PCA-SUP (Kiers 1991), es decir, el resultado de aplicar el Análisis de Componentes Principales (ACP) a la tabla global $X$ (o $Z$, según el caso).

A continuación se detallan los métodos que van a aplicarse, y que pasamos a describir brevemente.

Tabla 1: Técnicas aplicadas

\begin{tabular}{|c|c|}
\hline $\begin{array}{l}\text { Tablas } \\
\text { three-way } \\
\text { data array }\end{array}$ & $\begin{array}{l}\text { Métodos ICI: Asimétricos } \\
\text { - Statis (Statis y Statis dual) } \\
\text { - Análisis Factorial Múltiple (AFM) } \\
\text { Modelos de componentes: Simétricos } \\
\text { - Métodos de Tucker: TUCKALS3, TUCKALS2 } \\
\text { - PARAFAC/CANDECOMP } \\
\text { - PCA-SUP }\end{array}$ \\
\hline
\end{tabular}

\subsection{Descripción de métodos: Métodos Asimétricos}

Como se indica en Glaçon (1981: 22-26), nos encontramos ante técnicas en las que el objetivo principal es la definición de tipologías de objetos, o bien, el análisis de los tres modos. La primera etapa de estos métodos, la interestructura, tiene como objeto el estudio de la relación entre las tablas que forman la tabla global, a través de los operadores que las representan. La segunda, el compromiso, busca obtener una nube media (ponderada) que represente la estructura común de esas tablas $Z^{\mathrm{t}}$. El análisis de las relaciones entre individuos y variables en la tabla-compromiso es el objetivo de la tercera etapa (intraestructura).

\section{Statis}

Este método incluye dos versiones: la Statis, apropiada cuando los individuos son los elementos comunes en las $Z^{\mathrm{t}}$; y la Statis dual,

1 Existen múltiples modelos generados a partir de los primeros en la jerarquía, tales como el IDIOSCAL, INDSCAL, etc. 
cuando lo que es común son las variables. Por tanto, en las tablas threeway data array es posible aplicar ambas versiones del método.

La Metodología Statis fue desarrollada inicialmente por Escoufier, quién desarrolló la primera etapa del método en 1976; posteriormente L'Hermier des Plantes desarrolló el método completo (de la versión Statis) en su tesis doctoral (1976).

Cada tabla se identifica con $\left(Z^{\mathrm{t}}, M_{\mathrm{t}}, D\right)$ siendo $Z^{\mathrm{t}}$ la matriz de datos, $M$ la matriz diagonal que recoge el peso de las variables en cada tabla y $D$ la matriz diagonal de pesos de los individuos, que es común para todas las tablas.

En la versión Statis, en la interestructura, cada tabla está representada por la matriz de productos escalares entre individuos, el operador $W_{\mathrm{t}} D=Z^{\mathrm{t}} M\left(Z^{\mathrm{t}}\right)^{\prime} D$, que generalmente se divide entre su norma con el fin de equilibrar la influencia de todas las tablas en el análisis. El producto escalar entre dos operadores es una medida de la relación entre los grupos de variables que representan (coeficiente $R V$ ). La imagen euclídea de estos operadores permite determinar si dos tablas son semejantes o no, es decir, si los individuos que las componen se comportan o no de manera similar. En la versión Statis dual, el operador que representa a cada tabla es la matriz de correlaciones $V_{\mathrm{t}} M=\left(Z^{t}\right)^{\prime} D Z^{\mathrm{t}} M$ y la imagen euclídea de los mismos permite comparar el comportamiento de las variables en las tablas.

En la etapa denominada compromiso, el objetivo consiste en buscar la estructura común a las tablas, es decir, una nube "media" de individuos en la versión Statis, o una nube "media" de variables en la versión Statis dual. En el primer caso, el operador que representa esta nube (operador-compromiso $W D$ ) se define como media ponderada de los operadores $W_{t} D$, empleando como ponderación un $\alpha_{t}$ que se determina minimizando la distancia entre $W D$ y el resto de objetos. En la versión dual, el operador-compromiso $V M$ se define como media ponderada de los operadores $V_{\mathrm{t}} M$.

En la versión Statis, la intraestructura (tercera etapa) consiste en el estudio analítico de la nube compromiso de manera similar a un Análisis Componentes Principales: los vectores propios de $W D$ son las coordenadas de los $n$ individuos-compromiso en los ejes de inercia que originan los factores. Las coordenadas de las $\Sigma p_{\mathrm{t}}$ variables y sus correlaciones con esos factores se obtienen a través de las relaciones de transición. En el caso de la versión dual, los vectores propios del operador $V M$ son las 
coordenadas de las $p$ variables (variables-compromiso), empleando las relaciones de transición para obtener las coordenadas de los $\Sigma n_{\mathrm{t}}$ individuos.

El Statis se completa con la construcción de las trayectorias (cuanto $t$ representa el tiempo), que siempre ha sido la parte más controvertida de esta versión y que no vamos a describir. Sin embargo, para las tablas three-way array, el trazado de las trayectorias pueden hacerse empleando la versión dual, uniendo para cada individuo la coordenada que presenta en cada tabla $t$.

\section{Análisis factorial múltiple}

Este método ha sido desarrollado por Escofier y Pagès (1992) y se aplica, generalmente, en el tratamiento simultáneo de una serie de tablas con los mismos individuos pero iguales o diferentes variables.

De la misma manera que en el método Statis, se tiene que $n$ es el conjunto de individuos, $\Sigma p_{\mathrm{t}}$ el conjunto de variables y $T$ el conjunto de tablas. Además $p_{\mathrm{t}}$ es el conjunto de variables del grupo $t$ y $X^{t}$ (o $Z^{t}$ para variables centradas y estándarizadas) la tabla asociada. Para la aplicación del método se supone que los individuos y las variables tienen un peso: $p_{\mathrm{i}}(i=1 \ldots n)$ es el peso que afecta al individuo $i$, y la suma de los pesos es $1 ; m_{\mathrm{j}}$ es el peso asociado a la variable $x_{\mathrm{j}}\left(j \in p_{\mathrm{t}}\right)$. En este método se realizan análisis separados de los grupos de variables y el análisis conjunto, por lo que las ponderaciones de las variables son diferentes en cada caso. De este modo, tenemos las matrices diagonales de los pesos de individuos $D$, común para todas las tablas; $M$ para las variables y $M_{\mathrm{t}}$ para cada grupo $p_{t}$.

En AFM la primera fase es la intraestructura, y en ella se lleva a cabo un Análisis de Componentes Principales (ACP) en el que la influencia de cada grupo de variables está equilibrada (la ponderación que equilibra el papel de los grupos se consigue al dividir cada variable por el primer valor propio del ACP de la nube $t$ a la que pertenece la variable). El análisis que conlleva a la representación de los $n$ individuos-compromiso se realiza mediante un ACP de la tabla global $Z$, con las variables ponderadas. Además, el método permite realizar una representación superpuesta de las $T$ nubes de individuos. Así, aquellos individuos cuyos puntos parciales (los que representan a cada individuo en cada grupo) se sitúen próximos ilustran la estructura común de las distintas tablas 
analizadas; por el contrario, los que presenten puntos parciales alejados constituyen las excepciones a la estructura común.

En la interestructura, que es la segunda etapa en el AFM, se busca comparar globalmente los grupos de variables mediante su representación gráfica. Para ello, se toma como representación de cada tabla $t$ el operador $W_{\mathrm{t}} D$. Para realizar la representación, se considera que cada eje representa un factor del ACP de la etapa de intraestructura. Cada tabla se representa por un punto y la coordenada de $Z^{t}$ a lo largo de un eje es la inercia proyectada de las variables que lo forman.

\subsection{Descripción de métodos: Métodos simétricos}

Dentro de estos métodos, Tucker (1966: 279-311) introdujo un nuevo enfoque basado en la idea de que una estructura subyacente (factorial) estaba asociada a cada modo, y no hay un solo grupo de factores, sino tres. Es decir, los tres modos observados de los datos están asociados, cada uno, con otro modo (no observable) que puede considerarse como un conjunto de factores o categorías ideales. Los objetos, variables y ocasiones son considerados como combinaciones lineales de los objetos "idealizados", de las variables "latentes" y de las ocasiones "prototipo", respectivamente. Las relaciones entre los tres tipos de factores son consideradas en una tabla o matriz estimada por el modelo, denominada matriz core.

El inconveniente que se planteaba en este planteamiento es que los algoritmos desarrollados por Tucker no permitían obtener aproximaciones de los datos en el sentido de mínimos cuadrados, razón por la que se desarrollaron otros métodos. Así, Kroonenberg y De Leeuw desarrollaron dos métodos basados en el modelo original de Tucker, denominados TUCKALS3 y TUCKALS2, que sólo pueden aplicarse en tablas three-way array.

\section{TUCKALS3 y TUCKALS2}

Partimos de la tabla $Z=\left\{z_{\mathrm{ijt}}\right\}$. Aunque el objetivo de ambos métodos es descomponer o factorizar la matriz formada por los datos iniciales, el TUCKALS3 es más general en cuanto que ajusta el modelo original de Tucker en el sentido de mínimos cuadrados. Además, reduce los tres modos considerados, a diferencia del TUCKALS2 que sólo lo hace para los objetos y las variables. 
- El modelo TUCKALS3 se expresa de la forma siguiente:

$$
z_{\mathrm{ijt}}=\sum_{q=1}^{Q} \sum_{n=1}^{N} \sum_{r=1}^{R} a_{\mathrm{jq}} b_{\mathrm{in}} c_{\mathrm{tr}} g_{\mathrm{qnr}}
$$

- Y el TUCKALS2:

$$
z_{\mathrm{ijt}}=\sum_{q=1}^{Q} \sum_{n=1}^{N} a_{\mathrm{jq}} b_{\mathrm{in}} h_{\mathrm{qn}}
$$

La matriz $A$ (elementos $a_{\mathrm{jq}}$ ) es la matriz de componentes que corresponde a las variables, es decir, la formada por variables idealizadas; $B\left(b_{\text {in }}\right)$ es la relativa a las diferentes ocasiones y $C\left(c_{\text {tr }}\right)$ a los individuos.

Mientras que $I, P$ y $T$ representan, respectivamente, el conjunto de individuos, variables y ocasiones, $N, Q$ y $R$ expresan los respectivos conjuntos de componentes y, por tanto, $A$ es de orden $P \times Q$; $B$ es de orden $I \times N$ y el orden de $C$ es $T \times R$.

En el TUCKALS3 la matriz $G$ (matriz core) cuyos elementos son los valores $g_{\text {qnr }}$ representa el peso de una combinación específica de las componentes de los tres modos; en el TUCKALS2 la matriz $H$ (matriz core) se reduce a la combinación de dos modos.

En ambos métodos, el siguiente paso consiste en maximizar la inercia explicada a través del ajuste por mínimos cuadrados, de forma similar al Análisis de Componentes Principales.

Las dificultades planteadas en la interpretación de la tabla resultado en los modelos de Tucker originaron nuevos métodos basados en hipótesis más sencillas. Carroll y Chang, por un lado, y Harshman por otro, desarrollaron en 1970, y de manera independiente, un modelo que descomponía las tablas de tres entradas con los modos totalmente cruzados de una manera muy simple. Los primeros autores lo denominaron CANDECOMP (Canonical Decomposition) y Harshman lo denominó PARAFAC (Parallel Factor Analysis), y se describe a continuación.

\section{PARAFAC/CANDECOMP}

A diferencia de la concepción de Tucker, en este método solamente se determina un grupo de factores a partir de los datos observados que se puede interpretar mediante los tres modos de forma 
simultánea. El inconveniente radica en que los ejes se pueden interpretar en un único modo, ya que el método no permite rotaciones de los mismos.

Este método se puede considerar como un caso especial del TUCKALS2, en el sentido de que en éste los componentes se definen para las variables y los individuos, mientras que en PARAFAC/CANDECOMP se definen de forma simultánea para ambos.

Para la tabla de datos $Z$, el modelo sobre el que trabaja este método se expresa:

$$
\hat{z}_{\mathrm{ijt}}=\sum_{s=1}^{S} a_{\mathrm{js}} b_{\mathrm{is}} c_{\mathrm{ts}}
$$

siendo $a_{\mathrm{js}}, b_{\text {is }}$ y $c_{\mathrm{ts}}$ las coordenadas de las componentes para los individuos, variables y ocasiones, respectivamente, en la s-componente del método. La resolución del mismo, como en los anteriores consiste en maximizar la inercia explicada o bien minimizar:

$$
\sum_{i=1}^{n} \sum_{j=1}^{m} \sum_{k=1}^{p}\left(z_{\mathrm{ijk}}-\hat{z}_{\mathrm{ijk}}\right)^{2}
$$

\section{PCA-SUP}

Según Kiers (1991: 456), también es posible realizar, un Análisis de Componentes Principales (ACP) de las tablas yuxtapuestas $X^{1}$, $X^{2} \ldots X^{\mathrm{T}}$ (que se puede denominar PCA-SUP), y que considera que todas las tablas-ocasiones tienen la misma importancia. Por tanto, cada individuo está definido por un vector de $n T$ dimensiones y el análisis sólo es interesante si los datos son estables de unas ocasiones (tablas) a otras. Sin embargo, presenta el inconveniente de que no analiza el tercer modo.

A pesar de las diferencias metodológicas de los métodos tratados, se pueden encontrar elementos comunes. Así, según Kiers (1988: 16), el TUCKALS3 puede considerarse como una variante restringida de la primera fase de la metodología Statis (statis1, según Kiers 1991: 450) en la que se realiza un ACP de las matrices que recogen cada grupo de variables. Por otra parte, Bove y Di Ciaccio (1994) pusieron también de manifiesto las relaciones entre el método Statis y el modelo de Tucker2 (TUCKALS2). Tomando como referencia éstos y otros trabajos, se van a comparar los métodos descritos desde un punto de vista empírico, tomando como punto de partida los aspectos que analiza cada método, y los resultados que obtiene. Comprobaremos en el epígrafe 3 de este trabajo si dichas 
diferencias y semejanzas, se traducen o no en resultados empíricos diferentes.

\subsection{Comparación de métodos}

Teniendo en cuenta las características de cada método, presentamos un cuadro resumen con los aspectos que pueden analizarse con cada uno de ellos:

Tabla 2: Aspectos que analiza cada método

\begin{tabular}{|c|c|c|c|c|c|}
\hline \multirow{2}{*}{ Método } & $\begin{array}{c}\text { Comparación } \\
\text { de tablas } \mathbb{Z}^{\mathrm{t}}\end{array}$ & \multicolumn{2}{|c|}{ Variables } & \multicolumn{2}{c|}{ Individuos } \\
\cline { 4 - 6 } Statis & $\times$ & $\times$ & & & $\times$ \\
\hline Statis dual & $\times$ & & $\times$ & $\times$ & \\
\hline AFM & $\times$ & $\times$ & & $\times$ & $\times$ \\
\hline TUCKALS3 & $\times$ & & $\times$ & & $\times$ \\
\hline TUCKALS2 & & & $\times$ & & $\times$ \\
\hline PARAFAC/CANDECOMP & $\times$ & & $\times$ & & $\times$ \\
\hline PCA-SUP & & $\times$ & & & $\times$ \\
\hline
\end{tabular}

El Statis y el Statis dual son métodos que extraen una estructura común que resumen todas las tablas. En el caso del Statis, se extrae una estructura media-compromiso de individuos, quedando cada individuo-compromiso caracterizado según su posición en los planos formados por los factores que toman significado al relacionarse con las variables de todas las tablas (variables-parciales). En el caso del Statis dual, la nube media (o compromiso) de variables es una matriz de correlaciones que permite identificar factores definidos por las correlaciones con las variables-compromiso. La posición de cada individuo en cada tabla (individuo-parcial) se interpreta en función de su posición en los planos formados por esos factores, lo que permite el trazado de la trayectoria del individuo cuando cada tabla $t$ representa un instante temporal.

El método que considera más aspectos de la información es el AFM en cuanto que permite analizar las posiciones de los individuoscompromiso a través de unos factores que toman significado en función de sus correlaciones con las variables de cada tabla (variables-parciales). Además, el AFM permite caracterizar a cada individuo en cada tabla gracias a la representación simultánea de todos los individuos (individuos-parciales). Como en el Statis dual, este hecho permite el trazado de la trayectoria del individuo cuando las tablas se refieren a periodos de tiempo diferentes, 
con la ventaja de que con el AFM en la representación obtenida aparecen también las posiciones compromiso.

En el PCA-SUP, los factores identificados se obtienen en función de todas las variables de las tablas (sin ponderar) y cada individuo se caracteriza en función de su posición respecto a dichos factores. El inconveniente de este método es que no permite identificar relaciones entre las tablas, ya que todas las variables son tratadas por igual sin tener en cuenta la tabla de pertenencia.

Los anteriores métodos permiten relacionar fácilmente los planos de individuos y variables en cuanto que son métodos basados en el ACP, y por ello, las coordenadas (correlaciones) de las variables dan significado a los factores que determinan las posiciones de los individuos. No ocurre lo mismo con los métodos TUCKALS3, que identifican factores de forma independiente para tablas, variables e individuos, lo que permite describir por separado cada modo, siendo el elemento de conexión la mencionada matriz core. Esta matriz contiene los pesos de las combinaciones de los tres modos, es decir, la cantidad con la que cada una de las combinaciones contribuye a los datos, pero su interpretación resulta complicada a la hora de establecer relaciones entre individuos, variables y ocasiones. Lo mismo ocurre con el TUCKALS2, con el inconveniente añadido de que sólo identifica el modelo subyacente para dos modos (variables e individuos) dejado de lado las ocasiones y, por tanto, no permite comparar globalmente las tablas.

A diferencia de los dos anteriores, el modelo PARAFAC/CANDECOMP identifica un único sistema de factores para todo los modos, es decir, permite obtener tres matrices de componentes que resumen las relaciones lineales entre individuos, variables y ocasiones (no hay matriz core). Sin embargo, presenta el inconveniente de que el número de factores identificados en todos los modos tiene que ser el mismo, lo que limita considerablemente el método.

A continuación, se presentan detalladamente los resultados y ayudas a la interpretación que se obtienen con los métodos que analizan los tres modos, ya que los que analizan solamente los modos de individuos y variables (PCA-SUP y TUCKALS2) no permiten comparar globalmente las tablas $Z^{t}$. 
Tabla 3: Resultados y ayudas a la interpretación de cada método

\begin{tabular}{|c|c|c|c|c|c|c|c|c|}
\hline & \multirow[b]{2}{*}{ Factores } & \multirow[b]{2}{*}{ Aspectos analizados } & \multicolumn{6}{|c|}{ Resultados } \\
\hline & & & 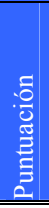 & 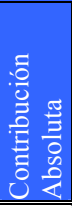 & $\begin{array}{l}0 \\
\text { o } \\
\frac{\pi}{3} \\
\frac{\pi}{5} \\
0 \\
0 \\
0 \\
0 \\
0 \\
0 \\
0 \\
\end{array}$ & 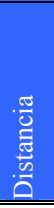 & $\begin{array}{l}\frac{0}{0} \\
\frac{0}{0} \\
\Sigma\end{array}$ & 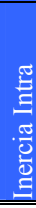 \\
\hline \multirow{4}{*}{ Statis } & \multirow{4}{*}{$\begin{array}{l}2 \text { sistemas de ejes: } \\
\text { diferentes para } \\
\text { Interestructura e } \\
\text { Intraestructura }\end{array}$} & Tablas $Z^{t}$ & $\times$ & $x$ & $x$ & $x$ & & \\
\hline & & Variable-parcial & $x$ & $\times$ & $x$ & & & \\
\hline & & Individuo-compromiso & $x$ & $x$ & $x$ & $x$ & & \\
\hline & & Individuo-parcial & & & & $\times$ & & \\
\hline \multirow{4}{*}{ Statis dual } & \multirow{4}{*}{$\begin{array}{l}2 \text { sistemas de ejes: } \\
\text { diferentes para } \\
\text { Interestructura e } \\
\text { Intraestructura }\end{array}$} & Tablas $Z^{t}$ & $x$ & $x$ & $x$ & $x$ & & \\
\hline & & Variable-parcial & & & & $x$ & & \\
\hline & & Variable-compromiso & $\times$ & $\times$ & $x$ & & & \\
\hline & & Individuo-parcial & $\times$ & $\times$ & $\times$ & & & \\
\hline \multirow{6}{*}{ AFM } & \multirow{6}{*}{$\begin{array}{l}1 \text { sistema de ejes: } \\
\text { el mismo para } \\
\text { Interestructura e } \\
\text { Intraestructura }\end{array}$} & Tablas $Z^{t}$ & $x$ & $x$ & $x$ & & & \\
\hline & & Variable-parcial & $\times$ & $\times$ & $\times$ & & & \\
\hline & & Individuo- compromiso & $x$ & $x$ & $x$ & $x$ & & \\
\hline & & Individuo-parcial & $x$ & $\times$ & $x$ & $x$ & & \\
\hline & & Ejes parciales & $\times$ & $x$ & $x$ & & & \\
\hline & & Individuo-parcial por factor & & & & & & $\times$ \\
\hline \multirow{3}{*}{ TUCKALS3 } & \multirow{3}{*}{$\begin{array}{l}3 \text { sistemas de ejes: } \\
\text { diferentes para } \\
\text { tablas, individuos y } \\
\text { variables }\end{array}$} & Tablas $Z$ & $x$ & & & & $x$ & \\
\hline & & Variable-compromiso & $x$ & & & & $\times$ & \\
\hline & & Individuo-compromiso & $\times$ & & & & $\times$ & \\
\hline \multirow{3}{*}{$\begin{array}{l}\text { PARAFAC/ } \\
\text { CANDECOMP }\end{array}$} & \multirow{3}{*}{$\begin{array}{c}1 \text { sistema de ejes: } \\
\text { el mismo para } \\
\text { tablas, individuos } \\
\text { y variables }\end{array}$} & Tablas $Z^{t}$ & 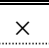 & & & & $x$ & \\
\hline & & Variable-compromiso & $\times$ & & & & $\times$ & \\
\hline & & Individuo-compromiso & $\times$ & & & & $\times$ & \\
\hline
\end{tabular}

Como puede comprobarse, el AFM es el método que permite analizar más detalladamente la tabla de tres entradas $Z$, seguido del Statis y del Statis dual. Los métodos TUCKALS3 y PARAFAC/CANDECOMP son los que obtienen un menor desglose de resultados aunque son los únicos que permiten extraer el modelo global de los datos.

Para todos los casos, éstos resultados numéricos se concretan y resumen en representaciones gráficas de tablas, individuos y variables, que dan una visión más general del fenómeno estudiado, y que son las que vamos a considerar en este trabajo dada la limitación de espacio.

\section{APLICACIÓN Y RESULTADOS}

Comenzamos la comparación empírica de métodos describiendo la recogida de información. Posteriormente se detallan los 
resultados obtenidos para los métodos Statis, Statis dual, AFM, TUCKALS3 y PARAFAC/CANDECOMP que son los que tratan los tres modos. Para aplicar estos métodos se han empleado dos programas estadísticos distintos: el SPAD v.6, para los tres primeros; y el módulo NWAY con Matlab7.1, para TUCKALS3 y PARAFAC/CANDECOMP.

\subsection{Recogida de información}

La información analizada en este trabajo se concreta en una tabla de tres entradas formada por las 93 empresas más grandes, según su cifra neta de negocio, en la Comunidad Autónoma de Castilla y León (individuos), sobre las que se han observado 18 indicadores económicofinancieros (variables), durante los años 2002, 2003 y 2004 (3 ocasionesaños-tablas). Esta información ha sido obtenida de la base de datos SABI, accesible a través de la Biblioteca de la Universidad de León. Las empresas analizadas se recogen en la tabla del Anexo. En cuanto a las variables, el detalle de cada una de ellas y su etiqueta se expone a continuación:

\section{Rentabilidad}

Rentabilidad económica o de los activos totales (ROA): El numerador del ratio lo componen los resultados después de impuestos y antes de intereses. El denominador está formado por el volumen de activos medios de la empresa durante el periodo de referencia. Mide la rentabilidad media de los activos de la empresa, al objeto de juzgar el grado de eficacia con que están siendo utilizados los recursos empleados, por lo que este ratio refleja la eficiencia operativa de la empresa.

Rentabilidad financiera o de los fondos propios (ROE): En el numerador aparecen los resultados después de impuestos y antes de intereses. El denominador lo integra la cifra media de fondos propios de la empresa durante el período analizado. Este ratio indica la rentabilidad del capital propiedad de los accionistas, y expresa cómo se traduce la eficiencia operativa en eficiencia para los accionistas.

\section{Gestión o actividad}

Margen Bruto (MBR): El numerador del ratio son los beneficios o pérdidas de explotación y en el denominador los ingresos de explotación. 
Mide la proporción de los resultados de explotación sobre el total de los ingresos por ventas.

Margen Comercial (MCO): En el numerador están los beneficios o pérdidas de explotación más los beneficios o pérdidas financieras, que se divide por los ingresos de explotación. Este ratio indica el porcentaje que suponen los beneficios por actividades ordinarias con relación al total de los ingresos por ventas.

Rotación de activos (RAC): Se define como el cociente entre los ingresos de explotación y los activos medios. Por consiguiente, mide la eficiencia de la empresa en el uso de los activos: tendrán un ratio superior aquellas empresas que requieran un volumen pequeño de activos para alcanzar una cifra elevada de ingresos de explotación.

Beneficio neto sobre ventas (RBV): Se calcula como el cociente entre el resultado del ejercicio y las ventas netas de la empresa. Por tanto, este ratio indica el porcentaje que supone el resultado neto de la empresa con relación al total de los ingresos por ventas.

Periodo medio de maduración de deudores (PMD): Este ratio se define como el cociente entre el volumen medio de clientes y las ventas diarias. Mide el número medio de días que se tardan en cobrar las cuentas de clientes.

Gastos financieros sobre ventas (GFV): Se calcula como el cociente entre la cifra de gastos financieros y los ingresos por ventas. Este indicador resume la proporción que suponen las cargas financieras sobre el importe neto de la cifra de negocios.

Depreciación sobre ventas (DVE): Este ratio se calcula como el cociente entre las dotaciones a la amortización del inmovilizado y los ingresos por ventas.

\section{Liquidez}

Ratio de circulante $(\mathrm{RCI})$ : Se calcula como el cociente entre la suma a todas las partidas del activo circulante y todas las partidas que componen el pasivo circulante. El ratio de circulante es un indicador de la solvencia a corto plazo de la empresa, revelando su capacidad para hacer frente a los pagos que van a demandarse en el siguiente ciclo de explotación. 
Prueba de ácido (PAC): El numerador se define como cuentas a cobrar, inversiones financieras temporales y tesorería, dividido todo ello por el pasivo circulante. Con respecto al anterior $(\mathrm{RCl})$, elimina el efecto distorsionador que pueda tener la aplicación de diferentes criterios de valoración de las existencias entre empresas.

Liquidez inmediata (LIQ): Al igual que el ratio de la prueba del ácido, deriva del ratio circulante. El numerador se define como la tesorería, mientras que el denominador se corresponde con el pasivo circulante.

Capital circulante sobre ventas (CCV): Este ratio se calcula como el cociente entre el capital circulante o fondo de maniobra (el fondo de maniobra se define como activo circulante menos pasivo circulante), y los ingresos por ventas. Por tanto, este ratio mide la cantidad de veces que el fondo de maniobra es superior a los ingresos por ventas.

\section{Estructura y solidez financiera}

Ratio de circulante sobre activo (RCA): Es el cociente entre el activo circulante y el activo total. Mide el peso que suponen las partidas más líquidas del activo con relación a su volumen total.

Neto sobre pasivo (NPA): El numerador de este ratio se define como los recursos o fondos propios de la empresa, y el denominador representa el pasivo total. Así, este ratio mide el porcentaje que suponen los recursos propios sobre el pasivo total.

Ratio de endeudamiento sobre pasivo (REP): Este ratio es complementario del anterior, en el sentido de que aquél mide la proporción que representan los fondos propios sobre el pasivo, y éste mide la proporción que representan los fondos ajenos. Por tanto, este ratio se calcula como el cociente entre los acreedores a corto y largo plazo y la cifra del pasivo total.

Ratio de endeudamiento a corto plazo (RECP): En el numerador aparece la cifra de acreedores a corto plazo, y en el denominador aparece la cifra de acreedores a corto y largo plazo. Por tanto, este ratio mide el porcentaje de la deuda que debe ser devuelta en un plazo no superior a un año.

Cobertura de las cargas financieras (CCF): Este ratio se define como el cociente entre el beneficio antes de intereses e impuestos, y los 
gastos financieros. Por tanto, este ratio mide la capacidad de la empresa para devolver, con base en su resultado de explotación, los intereses de la deuda.

Con el fin de identificar el año en el que está medida cada variable (o cada individuo), cada etiqueta se acompaña del dígito del año, según se haga referencia al 2002, 2003 ó 2004, respectivamente. La tabla de tres entradas $X$ resultante está formada por la yuxtaposición de 3 tablas de doble entrada $X^{2002}, X^{2003}, X^{2004}$, formada cada una por 93 filas (empresas) y 18 columnas (variables). Una vez centrada y estandarizada cada variable, la tabla de tres entradas resultante $(Z)$ tiene 93 filas y 54 columnas:

Figura 2: Tabla de tres entradas analizada

$$
\begin{array}{l|l|l|}
\hline Z^{2002} & Z^{2003} & Z^{2004} \\
\hline
\end{array}
$$

La tabla $Z$ será analizada por los métodos descritos en el apartado de metodología con un doble objetivo: comparar desde el punto de vista empírico los métodos, y describir la construcción en Castilla y León a través de sus mayores empresas en el período 2002-2004.

\subsection{Resultados}

Vamos a hacer referencia a los resultados obtenidos para cada uno de los tres modos: tablas (años), variables (ratios económico-financieros) e individuos (empresas). Para cada modo, los resultados se interpretan desde dos puntos de vista: en lo relativo al método aplicado, y en lo que se refiere a las empresas constructoras analizadas.

La gran cantidad de tablas y gráficos que se han obtenido superan los límites de este trabajo, motivo por el cuál sólo se presentan los más relevantes para efectuar la comparación entre los métodos, limitándonos a los gráficos para los tres factores de mayor inercia.

\subsubsection{Tablas (Años)}

Teniendo en cuenta lo señalado en el marco teórico, la comparación global de las tablas sólo se realiza con los métodos Statis, Statis dual, Análisis Factorial Múltiple, TUCKALS3 y PARAFAC/CANDECOMP. En primer lugar, presentamos la tabla de inercia con 2 y 3 factores: 
Tabla 4: Inercias de los factores para las tablas

\begin{tabular}{|c|c|c|}
\hline Método & $\begin{array}{c}\text { 2 factores } \\
\text { \% inercia }\end{array}$ & $\begin{array}{c}\text { 3 factores } \\
\text { \% inercia }\end{array}$ \\
\hline Statis & 81,42 & 100,00 \\
\hline Statis dual & 94,33 & 100,00 \\
\hline AFM & 35,26 & 44,41 \\
\hline TUCKALS3 & 32,89 & 41,44 \\
\hline PARAFAC/CANDECOMP & 32,76 & 41,26 \\
\hline
\end{tabular}

Como puede comprobarse, los métodos que explican un mayor porcentaje de información son el Statis y el Statis dual. Además, en ambos métodos se alcanza una calidad de representación del $100 \%$ con tres factores, siendo con dos factores superior al $80 \%$.

En el caso del AFM, la igualdad en los factores de las dos etapas se debe a que el análisis global de las tablas (interestructura) está condicionada por el análisis de los individuos y de las variables (intraestructura). Como consecuencia, y frente al Statis, cada eje obtenido para comparar las tablas puede interpretarse en función de los grupos de variables con los que está relacionado; sin embargo, la calidad global de la representación no es perfecta en la mayoría de las ocasiones, aunque se conserven todos los ejes (con tres factores sólo se explica el $44,41 \%$ de la información).

En el caso de los métodos TUCKALS3 y PARAFAC/CANDECOMP, las inercias obtenidas coinciden en todos los modos dado que son métodos simétricos que tratan todos ellos por igual. Como se observa en la tabla, la representación obtenida no sólo es de menor inercia que las del Statis, Statis dual y AFM, sino que los planos obtenidos tampoco pueden interpretarse directamente en función de variables e individuos: en TUCKALS3 porque hay tres grupos de factores diferentes (uno para cada modo) y se relacionan a través de la matriz core de difícil interpretación; en PARAFAC/CANDECOMP porque la interpretación es poco intuitiva, dado que los modos no cuentan con ayudas a la interpretación y queda limitado a sus puntuaciones, interpretando los gráficos en función de posiciones y distancias.

Por tanto, en la comparación global de tablas, el criterio del Statis y del Statis dual se impone por razones de inercia y por la calidad óptima de la representación global. Además, el primer eje es combinación lineal de los elementos representativos de cada tabla, y por tanto, interpretable en tanto que constituye el compromiso o nube media (de 
individuos en el Statis y de variables en el Statis dual). Comenzamos tomando las representaciones de estos métodos para obtener conclusiones respecto a la construcción en Castilla y León.

Sobre las empresas analizadas en el periodo

- Dado el porcentaje de inercia elevado, para Statis y Statis dual nos limitamos al primer plano factorial (plano 1-2), formado por los factores 1 y 2.

Figura 3: Plano factorial 1-2 para las tablas: métodos Statis y Statis dual, respectivamente
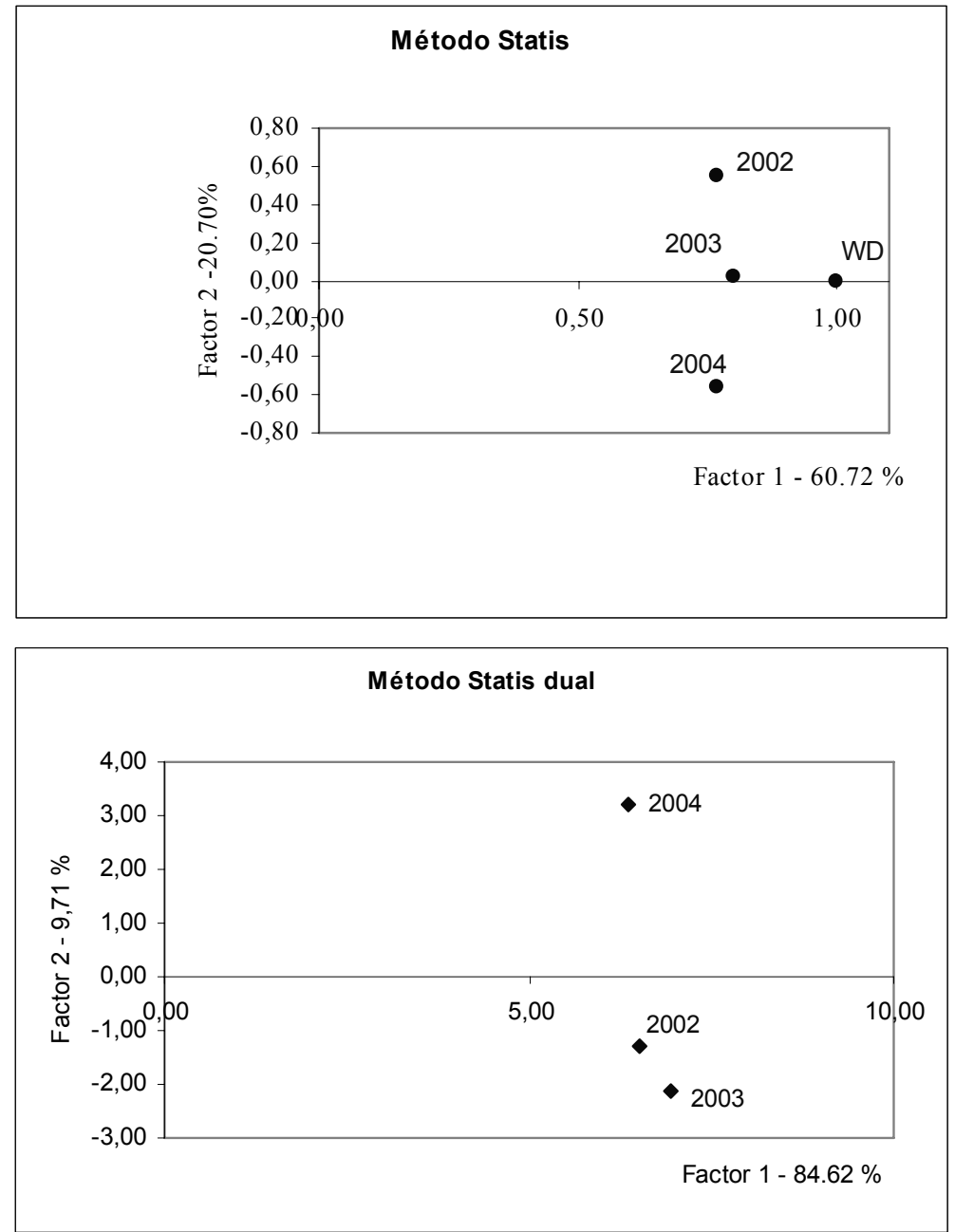
Como se deduce, el porcentaje de inercia del plano que se obtiene con el Statis es del $81,42 \%$ y con el Statis dual es del $94,33 \%$. En uno y otro caso, las tres tablas toman valores similares respecto al primer factor, lo que permite afirmar que las tres tablas definen nubes que pueden analizarse conjuntamente (tanto de individuos como de variables). En cuanto a la imagen del Statis, el cambio de posición respecto al segundo factor se interpreta como un cambio en el comportamiento de las empresas observadas: se produce una evolución en el sentido descendente de dicho eje conforme avanza el tiempo, siendo la trayectoria entre el 2002 y 2003 similar a la que marcan el año 2003 y 2004. También puede observarse como la nube más próxima a la nube media (o compromiso) es la del año 2003.

En cuanto a la imagen del Statis dual, la mayor inercia de los factores se debe a que las nubes de variables son más parecidas entre sí que las nubes de individuos. En este caso, la nube relativa al 2002 es la que más se asemeja al compromiso o nube media de variables. El ángulo que forman los años 2002 y 2003 es más pequeño que el que forman los años 2003 y 2004, lo que indica un mayor cambio en las variables correspondientes a estos últimos años.

Conviene precisar que para Statis y para Statis dual, los factores de esta etapa no son interpretables directamente en función de individuos y las variables, y sólo expresan los cambios acontecidos.

- Aunque la calidad de representación es menor, la imagen de las tablas obtenida con el AFM permite completar los resultados anteriores. Presentamos dos planos factoriales (plano 1-2 y plano 1-3), que sólo agrupan el $44,41 \%$ de la información, lejos del porcentaje alcanzando con uno sólo plano factorial de los dos métodos anteriores.

Las tres tablas toman valores similares respecto al primer factor, por lo que el cambio en las tablas se presenta sobre el segundo y tercer factores. Con respecto al segundo factor, el mayor cambio se produce entre los años 2003 y 2004, quedando en una posición intermedia en el año 2002. El cambio en el tercer factor se manifiesta en todo el periodo: entre los años 2002 y 2003, por un lado; y entre los años 2003 y 2004, por el otro. A diferencia de lo que ocurre con Statis y Statis dual, estos factores son los mismos que en la intraestructura, y por tanto, interpretables directamente en función de variables e individuos. 
Figura 4: Imagen de las tablas con AFM: plano 1-2 y plano 1-3, respectivamente
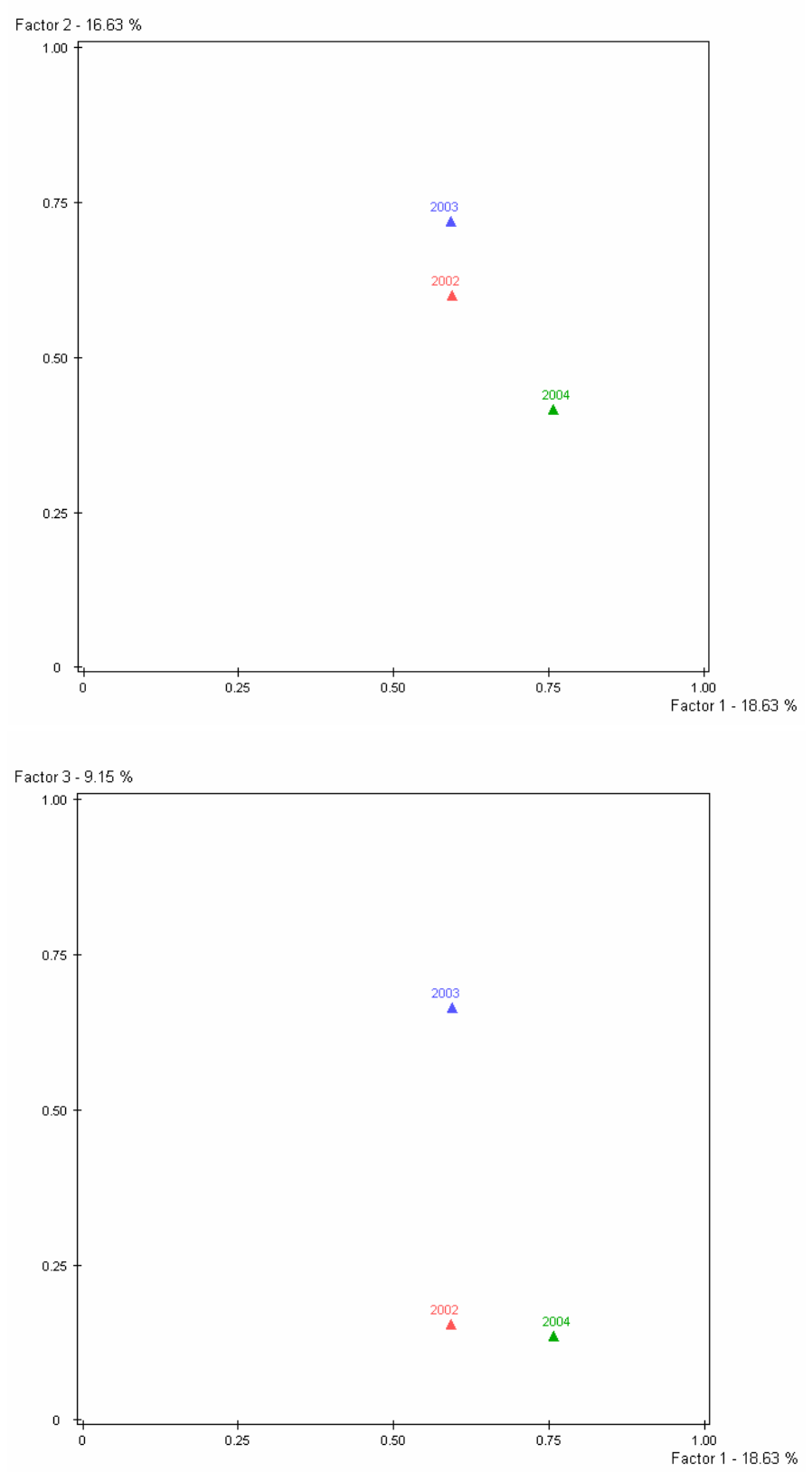

- A continuación, presentamos únicamente la representación gráfica obtenida con el método TUCKALS3 puesto que con el método PARAFAC/CANDECOMP se obtiene una representación similar. 
Figura 5: Imagen de las tablas con PARAFAC/CANDECOMP: plano 1-2 y plano 1-3, respectivamente

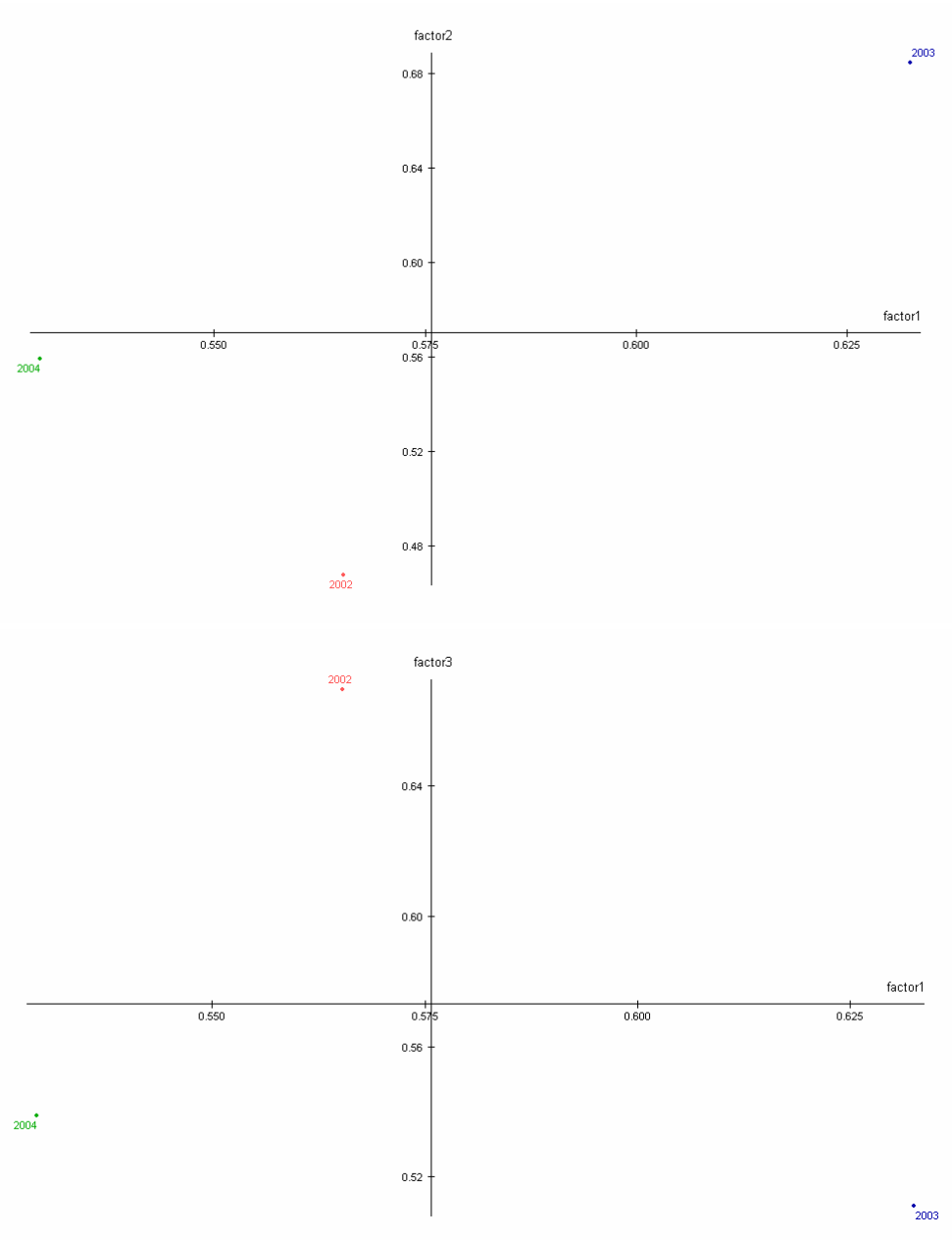

Como puede comprobarse, el año 2003 aparece en un posición opuesta a los años 2002 y 2004 (en ambos planos), lo que indica que los individuos y/o las variables que forman parte de las tablas han cambiado en ese periodo. Conviene señalar que también hay diferencias entre los años 2002 y 2004 ya que aunque están en el mismo cuadrante del plano 1-2, no ocupan la misma posición respecto al factor 2 ni tampoco respecto al factor 3 (plano 1-3).

Estas diferencias entre los años tiene una difícil interpretación ya que este método no permite (tampoco el PARAFAC/ CANDECOMP) obtener planos con individuos o variables desglosados por años. 
Finalizado el análisis de las tablas, y como paso previo al análisis de individuos y variables, se presenta la inercia explicada por los tres primeros factores de los métodos que analizan los tres modos.

\subsubsection{Inercia explicada}

La tabla 5 presenta la inercia extraída por cada método.

Tabla 5: Inercias de los factores (variables-individuos)

\begin{tabular}{|c|c|c|c|}
\hline Método & $\begin{array}{c}\text { 1 factor } \\
\text { \% inercia }\end{array}$ & $\begin{array}{c}\text { 2 factores } \\
\text { \% inercia } \\
\text { acumulada }\end{array}$ & $\begin{array}{c}\text { 3 factores } \\
\text { \% inercia } \\
\text { acumulada }\end{array}$ \\
\hline Statis & 18,70 & 35,72 & 44,58 \\
\hline Statis dual & 23,88 & 44,46 & 55,75 \\
\hline AFM & 18,63 & 35,26 & 44,41 \\
\hline TUCKALS3 & 17,12 & 32,89 & 41,44 \\
\hline PARAFAC/CANDECOMP & 17,22 & 32,76 & 41,26 \\
\hline
\end{tabular}

Como ya se ha indicado, la inercia de los factores para los métodos AFM, TUCKALS3 y PARAFAC/CANDECOMP es la misma que la ya presentada en la tabla 4. El método que extrae una mayor inercia es el Statis dual, seguido del Statis, aunque no hay grandes diferencias entre este último y el AFM.

Vamos a considerar los planos factoriales obtenidos con cada técnica, para variables e individuos, haciendo referencia a los resultados que se deducen en cada caso sobre la información analizada.

\subsubsection{Variables (Ratios económico-financieros)}

- Los planos formados por los factores 1 y 2 (plano 1-2) y los factores 1 y 3 (plano 1-3) son muy similares en el Statis y en el AFM. Las variables próximas al círculo de correlaciones estarán bien representadas y los vectores que unen el origen a los puntos-variable permiten visualizar los ángulos entre variables y medir la relación entre ellas.

Como se señala en la tabla 3 , ambos métodos definen los factores en función de la correlación de las variables de todas las tablas, lo que permite detectar los cambios de unos años a otros. Así, cuando para un factor determinado la correlación de una variable para los 3 años sea similar, se deduce que la variable presenta un comportamiento estable en 
el periodo estudiado. Por el contrario, las variables con correlación diferente según el año reflejan los cambios del periodo.

Figura 6: Imagen de las variables del Statis: plano 1-2 y plano 1-3, respectivamente.
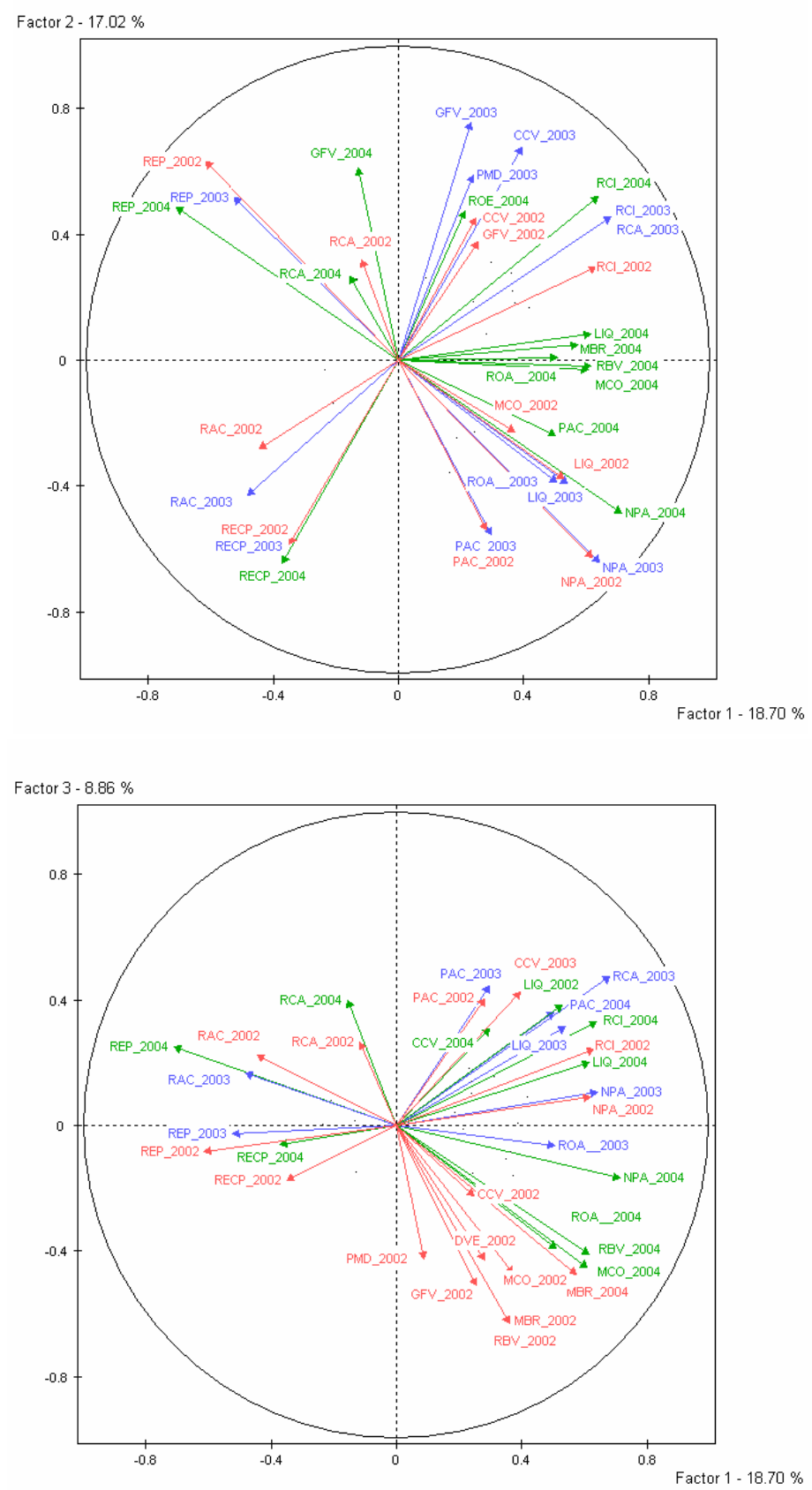


\section{Sobre las empresas analizadas en el periodo}

Considerando la imagen del Statis (figura 6) y la tabla 2 del Anexo, el factor 1 se identifica, en la parte positiva, con las variables relativas al neto sobre el pasivo en los tres años (NPA_2002, NPA_2003, NPA_2004), a la liquidez inmediata (LIQ 2002, LIQ_2003 y LIQ 2004), y al ratio de circulante (RCl_2002, RCI_2003 y RCl_2004) ya que son las que presentan una correlación más alta con este lado del factor. Por el lado negativo, el ratio de endeudamiento sobre pasivo es el que define el factor (REP_2002, REP_2003 y REP_2004).

Por tanto, el factor 1 puede denominarse ESTRUCTURA DEL PASIVO y establece una clara relación en el sector: las empresas con mayor proporción de neto sobre el pasivo son las que presentan una mayor liquidez a corto plazo; por el contrario, en aquellas en las que el ratio de endeudamiento sobre el pasivo es más elevado, el ratio de liquidez es bajo respecto a la media. Además, en el periodo estudiado hay una clara vinculación entre la mayor capacidad de la empresa para hacer frente a los pagos del siguiente ciclo de explotación y la mayor disponibilidad de tesorería, teniendo en cuenta las correlaciones señaladas entre $\mathrm{RCI}$ y LIQ. Por otra parte, todas las variables mencionadas son bastante estables en el periodo, ya que apenas hay diferencias de unos años a otros.

La parte positiva del factor también esta correlacionada con las variables de gestión relativas al año 2004, como son el margen bruto (MBR_2004), el margen comercial (MCO_2004), beneficio sobre ventas (RBV_2004) y la rentabilidad de los activos totales (ROA_2004). Por el lado negativo, el factor también presenta una correlación alta con la variable rotación de activos en el 2002 y 2003 (RAC_2002, RAC_2003).

Estos hechos dan lugar a interpretaciones diferentes en lo relativo al año 2004 y que explicarían por qué en la figura 4 la tabla que lo representa esté situada más a la derecha del factor 1 . Para este año, se da una clara vinculación entre neto sobre el pasivo, liquidez y mayor actividad de la empresa. También hay relación entre la rentabilidad de los activos totales y el margen comercial, que no se da entre los años 2002 y 2003. Además, la rotación de activos parece estar relacionada con el endeudamiento a corto plazo en los años 2002 y 2003, pero no en el 2004.

El factor 2 se correlaciona principalmente con el ratio de endeudamiento a corto plazo para los 3 años (RECP_2002, RECP_2003, RECP_2004) en la parte negativa. Por tanto, podemos identificar este segundo factor como ENDEUDAMIENTO A CORTO PLAZO. Con la parte positiva, 
aunque en menor intensidad, se correlaciona con el ratio que mide la proporción de gastos financieros sobre ventas para los 3 años (GFV_2002, GFV_2003 y GFV_2004), con las variables de capital circulante sobre ventas del año 2002 y 2003 (CCV_2002, CCV_2003), con el ratio de circulante sobre total de activo del año 2003 (RCA_2003), con el ratio de circulante del 2003 (RCl_2003) y con la rentabilidad económica del 2004 (ROE_2004). En el lado negativo, están correlacionadas la prueba de acido de los años 2002 y 2003 (PAC_2002, PAC_2003).

Los gastos financieros sobre ventas parece que tienen un comportamiento estable en el periodo, lo que no es de extrañar puesto que también es estable el nivel de endeudamiento. Además, hay una clara relación entre el capital circulante sobre ventas de los años 2002 y 2003 y los gastos financieros en el periodo, cuestión que cambia en el año 2004; y entre el endeudamiento a corto plazo y la prueba de acido en el año 2002 y 2003, que también varía en el año 2004. Como consecuencia, y como muestra la figura 4, la tabla correspondiente al año 2004 desciende respecto al factor 2 .

La parte positiva del factor 3 no se correlaciona de manera significativa con ninguna de las variables analizadas. En cuanto a la parte negativa, las variables con mayor correlación son las del 2002: margen bruto de ventas (MBR_2002), ratio de beneficio sobre ventas (RBV_2002), margen comercial (MCO_2002); y las variables del año 2004 margen bruto de ventas (MBR_2004), margen comercial (MCO_2004), y rentabilidad económica (ROA_2004). Por tanto, es el factor de ACTIVIDAD que refleja similitud entre los años 2002 y 2004. Este hecho puede explicar la proximidad entre las tablas de esos años en la imagen del AFM del factor 3 (figura 4), y la lejanía de la tabla 2003.

\subsubsection{Variables-compromiso}

- Las nubes medias de las variables se presentan por separado para el Statis dual y para los métodos TUCKALS3 y PARAFAC/ CANDECOMP, ya que en el primer caso (figura 7), se trata de correlaciones de variables-factor, y en el segundo caso (figura 8 y figura 9), se trata de puntuaciones que se interpretan en términos de distancias, alejamientos y proximidades. 
Figura 7: Imagen variables-compromiso con el Statis dual: plano 1-2 y plano 1-3, respectivamente
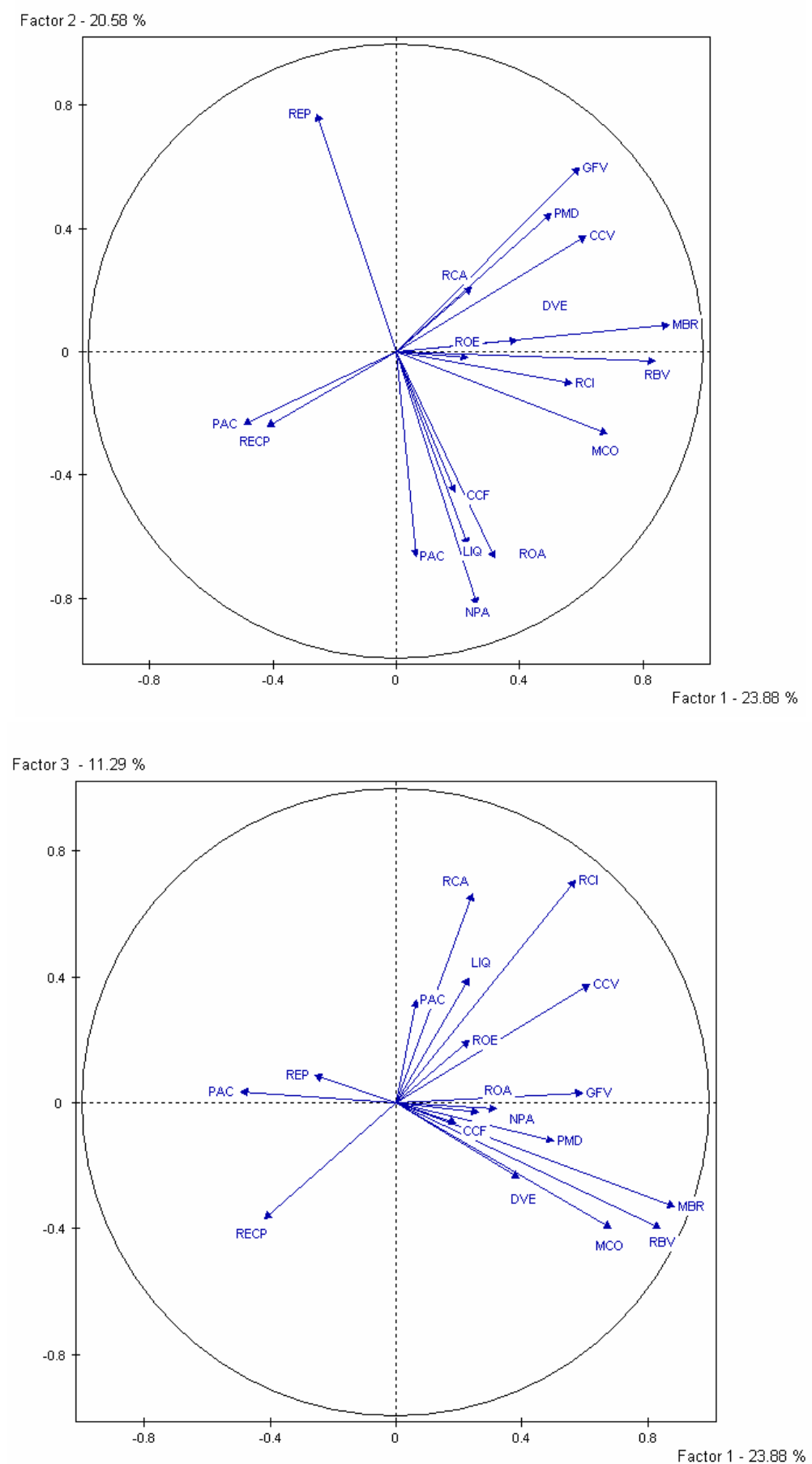
Como se ha indicado, en Statis y AFM la correlación similar con los factores de una variable en todos los años se interpreta como estabilidad en el periodo. Precisamente, para las variables estables la imagen de su variable-compromiso representada por el Statis dual presenta una posición y una correlación similares a las variables-parciales. Sin embargo, las variables-compromiso relativas a las variables con cambios en el periodo ocupan una posición intermedia, lo que en algunos casos provoca una modificación en el significado de los factores. Además, y como vamos a ver, la imagen de las variables-compromiso obtenidas con este método no permite detectar los cambios en el periodo, y la interpretación de los factores debe ser más cautelosas ya que se les asigna significado según la correlación "media" de las variables-compromiso.

Sobre las empresas analizadas en el periodo

Con el fin de extraer conclusiones relativas a la información analizada, pasamos a comentar los diferentes factores.

La parte positiva del factor 1, está correlacionada principalmente con las variables de actividad: margen bruto sobre ventas (MBR), beneficio neto sobre ventas (RBV), margen comercial (MCO), y gastos financieros sobre ventas (GFV). También se correlaciona con el lado positivo la variable capital circulante sobre ventas (CCV). En la parte negativa, las variables con alta correlación son la rotación de activos (RAC) y el ratio de endeudamiento a corto plazo (RECP). Por tanto, se puede afirmar que el factor 1 es un factor de ACTIVIDAD.

Por tanto, en el periodo considerado, hay relación entre la mayor actividad de la empresa y el capital circulante, por un lado; y entre la rotación de activos y el endeudamiento a corto plazo, por el otro.

El factor 2 está correlacionado en la parte positiva con el ratio de endeudamiento sobre pasivo (REP), y en el lado negativo con el neto sobre el pasivo (NPA). Por otra parte, la prueba del ácido (PAC), la liquidez (LIQ), y la rentabilidad económica (ROA) también están correlacionadas con la parte negativa del factor. El factor 2 es identificado como ESTRUCTURA DE PASIVO.

Este factor indica que, con carácter general en el sector, mayor neto patrimonial implica mayor rentabilidad de los activos y mayor liquidez. 
El factor 3 está correlacionado en la parte positiva con el ratio de circulante sobre activo (RCA) y con el ratio de liquidez $(\mathrm{RCl})$. Por lo que el factor 3 se denomina LIQUIDEZ, y vincula mayor activo circulante con mayor liquidez.

Por tanto, respecto a Statis y AFM, el Statis dual extrae factores similares, aunque con un significado más general.

- Presentamos la imagen en los planos 1-2 y 1-3 de las variables-compromiso con el método TUCKALS3.

En este caso no es posible dotar de significado a los factores, ya que sólo se obtienen puntuaciones de las variables y no correlaciones ni contribuciones absolutas (tabla 3). Por tanto, sólo se extraen conclusiones en términos de diferencias y semejanzas para el periodo considerado entre las variables-compromiso, ya que tampoco se representan con estos métodos las variables parciales.

Figura 8: Imagen variables-compromiso TUCKALS3 : plano 1-2 y plano 1-3, respectivamente

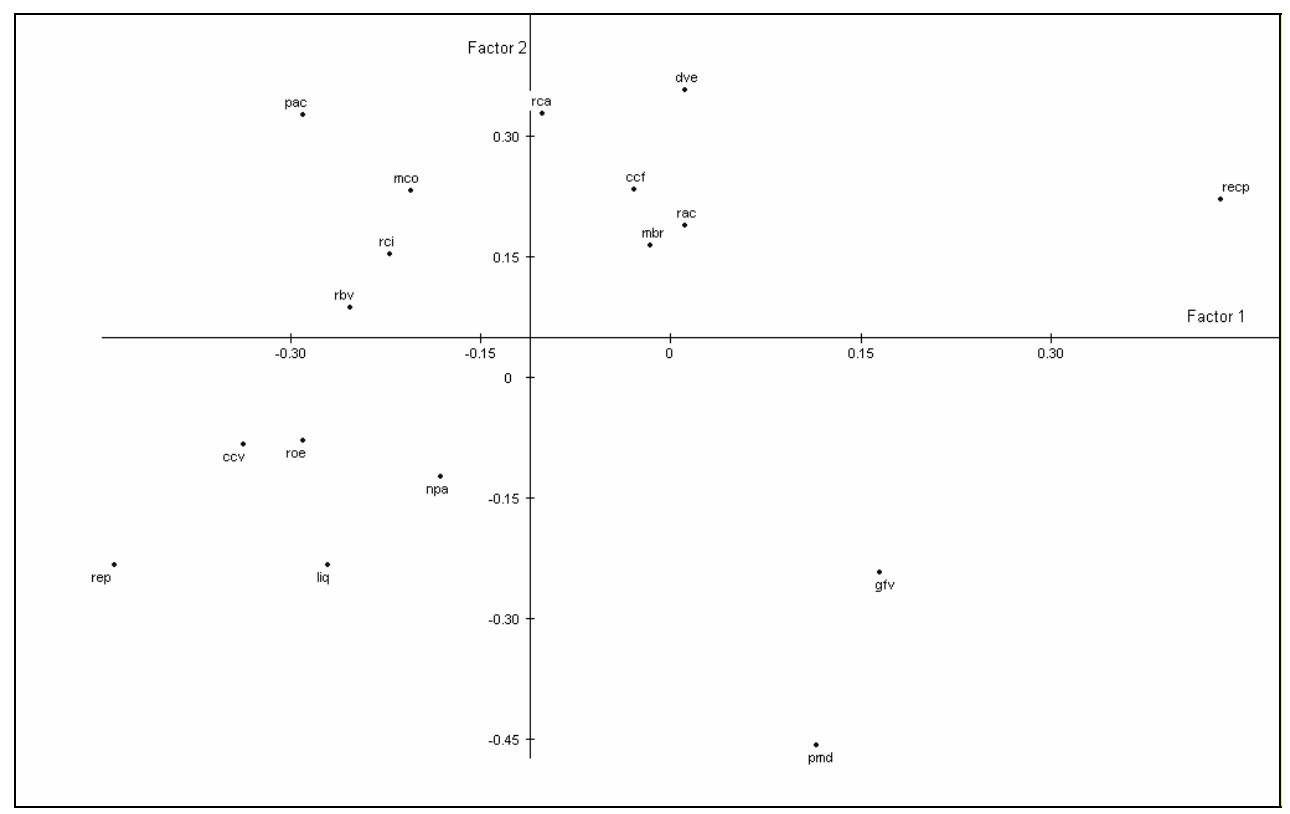




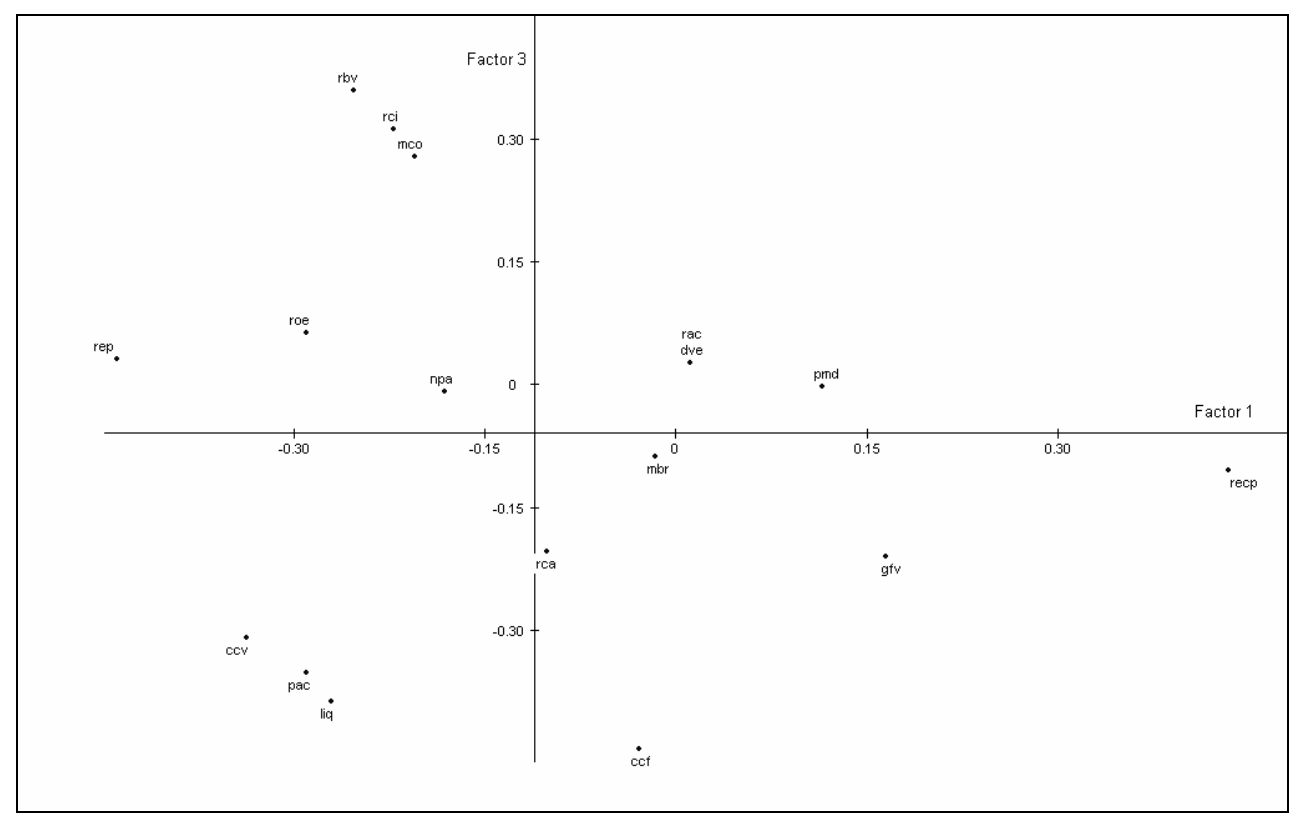

Sobre las empresas analizadas en el periodo

Sin ser exhaustivos, y limitándonos a las posiciones más extremas, en el primer cuadrante del plano 1-2 destaca la variable endeudamiento a corto plazo sobre el pasivo (RECP); en el segundo cuadrante la variable prueba de acido (PAC); en el tercer cuadrante, ratio de endeudamiento sobre pasivo (REP), y en el cuarto gastos financieros sobre ventas (GFV) y periodo medio de maduración de deudores (PMD).

En el plano 1-3, en el segundo cuadrante destacan tres variables: ratio de beneficio sobre ventas $(\mathrm{RBV})$, ratio de circulante $(\mathrm{RCl})$ y margen comercial (MCO). En el tercer cuadrante de este plano destacan las variables capital circulante (CCV), prueba de ácido (PAC) y liquidez (LIQ).

- A continuación, presentamos los planos factoriales de las variables con el método PARAFAC/CANDECOMP. 
Figura 9: Imagen variables-compromiso PARAFAC/CANDECOMP: plano 1-2 y plano 1-3, respectivamente

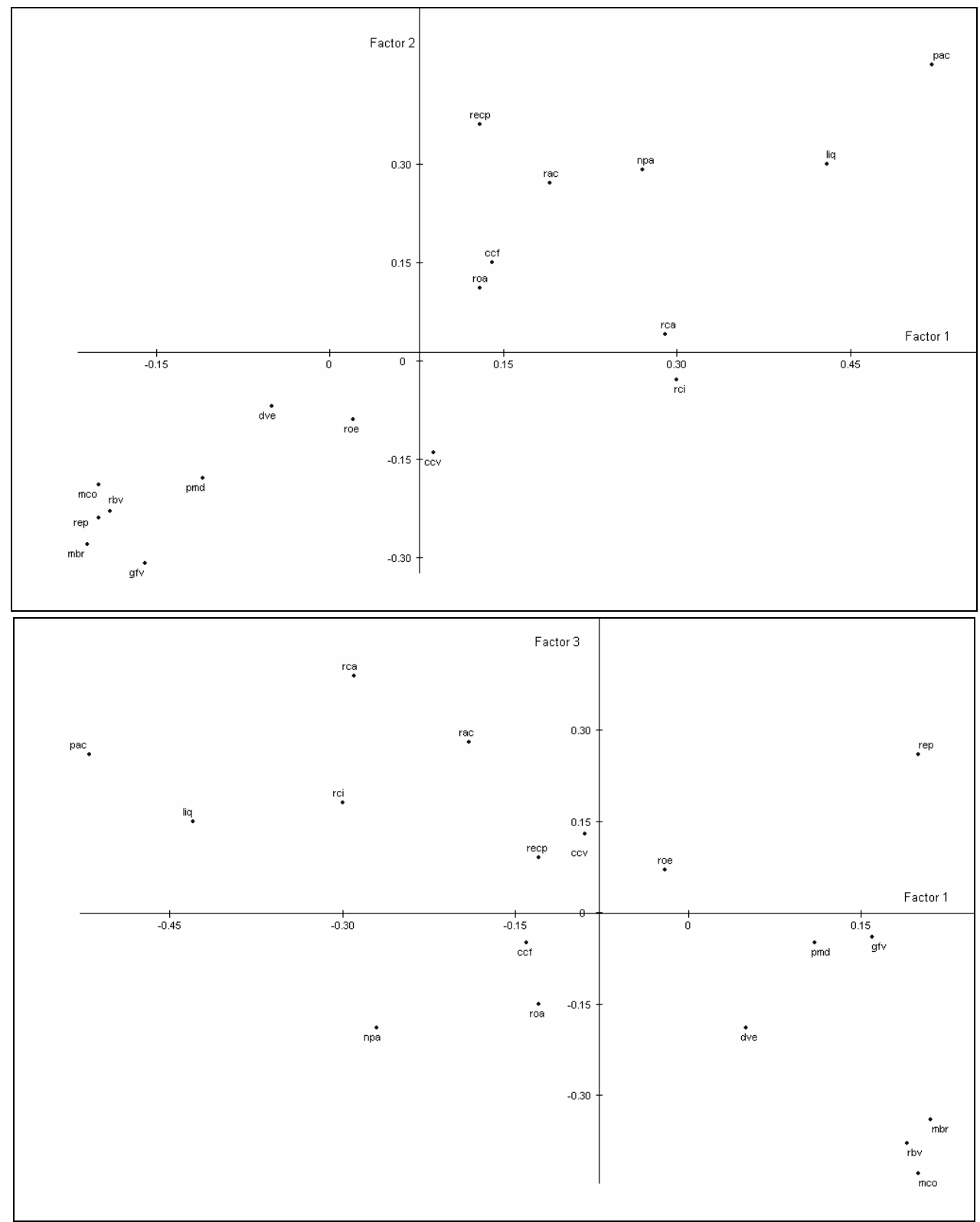

Sobre las empresas analizadas en el periodo

Al interpretar la figura 9, obtenemos conclusiones similares en términos de proximidades y distancias a las ya comentadas con la 
imagen de TUCKALS3. Citando solamente las posiciones destacadas, en el primer cuadrante del plano 1-2 aparecen las variables liquidez (LIQ) y prueba de ácido (PAC), aunque también se encuentra en el mismo el neto sobre el pasivo (NPA), el ratio de endeudamiento a corto plazo (RECP) y el ratio de activo (RAC). Por otra parte, en el mismo plano, pero en el cuadrante opuesto, se presentan algunas variables de actividad, tales como el margen bruto (MBR), margen comercial (MCO), beneficio sobre ventas (RBV), gastos financieros sobre ventas (GFV), y todas ellas relativamente próximas al periodo medio de maduración de deudores (PMD).

En el plano 1-3, la variable de endeudamiento destaca en el primer cuadrante; las variables de liquidez en el segundo; la variable de neto sobre el pasivo (NPA) en el tercero; y las variables de actividad margen bruto sobre ventas (MBR), margen comercial (MCO), y beneficio neto sobre ventas (RBV) en el cuarto cuadrante.

Como puede comprobarse, tanto TUCKALS3 como PARAFAC/ CANDECOMP sólo extraen conclusiones en términos de diferencias y semejanzas entre variables-compromiso para el periodo considerado, que básicamente coincide con las extraídas por otros métodos.

\subsubsection{Individuos-compromiso}

Pasamos a considerar los dos planos factoriales para los individuos, comenzando por las posiciones compromiso del periodo que se obtienen con los métodos Statis, AFM, TUCKALS3 y PARAFAC/CANDECOMP.

- En primer lugar, se presenta la imagen del Statis, que es muy similar a la imagen de individuos-compromiso obtenida con el AFM, por lo que dejaremos esta última para analizar las trayectorias de los individuos. 
Figura 10: Imagen de los individuos-compromiso obtenida con el Statis: plano 1-2 y plano 1-3, respectivamente
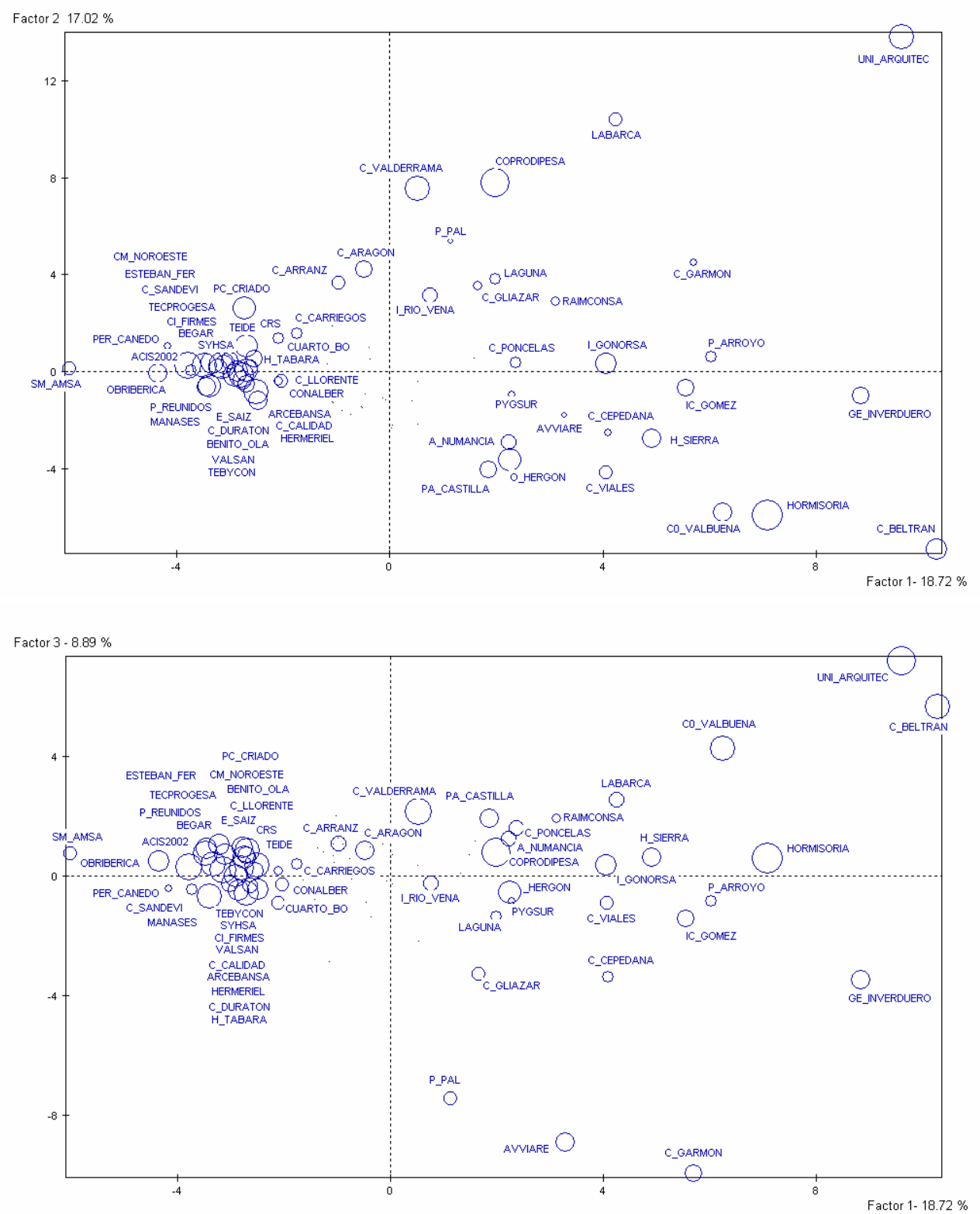

Los dos métodos permiten representar las posiciones de los individuos en función de la calidad de representación en el plano (los que tienen icono más grande están mejor representados). Para interpretar 
correctamente los planos factoriales, es necesario hacer algunas apreciaciones:

- Los individuos cuya representación está cerca del origen (que es el centro de gravedad) tienen una difícil interpretación. Éstos no presentan valores muy extremos para el conjunto de variables, sino más bien en torno a los valores medios de todas ellas. Estos individuos no suelen representarse, como en este caso, puesto que resultarían unos planos factoriales poco legibles.

- Los que ocupan posiciones más extremas son los que tienen un comportamiento más destacado, y cuya posición se interpreta teniendo en cuenta el significado de los factores. Es decir, interesa el movimiento de los individuos en la dirección de las variables, por lo que un individuo estará en la dirección de las variables para las que tiene valores altos y en el lado opuesto de las variables con valores bajos. Por otra parte, si una variable está muy correlacionada con un factor significa que los individuos que poseen una coordenada elevada positiva con él están caracterizados por valores de la variable muy superiores a la media.

- También hay individuos bien representados, aunque en una posición menos destacada y que deben analizarse teniendo en cuenta que las características presentadas están menos acentuadas.

- Los individuos en posición destacada y que no están bien representados, se debe a que los correspondientes individuosparciales tienen posiciones alejadas entre sí. La trayectoria de estos individuos es la que interesa analizar posteriormente.

\section{Sobre las empresas analizadas en el periodo}

De la combinación de todos los criterios, obtenemos la tabla 6 que resume la posición de los individuos-compromiso destacados (cuyo nombre completo aparece en la tabla 1 del anexo) y su significado a la hora de extraer conclusiones para el sector. Las empresas constructoras que no se presentan en la tabla reflejarían un comportamiento cercano a la media para la mayoría de las variables estudiadas en el periodo 20022004. 
Tabla 6: Posición de los individuos-compromiso más destacados: características

\begin{tabular}{|c|c|c|c|c|}
\hline $\begin{array}{l}\text { Situación } \\
\text { en los } \\
\text { planos }\end{array}$ & $\begin{array}{l}\text { Posición } \\
\text { Destacada }\end{array}$ & $\begin{array}{l}\text { Posición } \\
\text { Moderada }\end{array}$ & $\begin{array}{l}\text { Posición } \\
\text { destacada } \\
\text { pero mal } \\
\text { representados } \\
\text { (Trayectoria) } \\
\end{array}$ & Características \\
\hline Factor $1+$ & GE_INVERDUERO & $\begin{array}{l}\text { IC_GOMEZ } \\
\text { H_SIERRA } \\
\text { I_GONORSA } \\
\text { C_VIALES }\end{array}$ & $\begin{array}{c}\text { C_CEPEDANA } \\
\text { P_ARROYO }\end{array}$ & $\begin{array}{l}\text { Neto sobre pasivo alto } \\
\text { Liquidez inmediata y ratio de } \\
\text { circulante altos } \\
\text { Endeudamiento bajo } \\
\text { Márgenes elevados en } 2004\end{array}$ \\
\hline $\begin{array}{l}\text { Factor } 1+ \\
\text { Factor 2+ }\end{array}$ & UNI-ARQUITEC & & C_GARMON & $\begin{array}{l}\text { Liquidez inmediata y ratio de } \\
\text { circulante altos } \\
\text { Endeudamiento bajo } \\
\text { Gastos financieros sobre ventas } \\
\text { altos }\end{array}$ \\
\hline Factor $2+$ & $\begin{array}{l}\text { C_VALDERRAMA } \\
\text { COPRODIPESA }\end{array}$ & $\begin{array}{l}\text { C_ARAGON } \\
\text { I_RIO_VENA }\end{array}$ & $\begin{array}{l}\text { LAGUNA } \\
\text { P_PAL } \\
\text { LABARCA }\end{array}$ & $\begin{array}{l}\text { Gastos financieros sobre ventas } \\
\text { altos } \\
\text { Endeudamiento a corto plazo bajo } \\
\text { Prueba ácido } 2002 \text { y } 2003 \text { baja } \\
\end{array}$ \\
\hline Factor 1 - & $\begin{array}{l}\text { SM_AMSA } \\
\text { OBRIBERICA }\end{array}$ & $\begin{array}{c}\text { ACIS_2002 } \\
\text { BEGAR } \\
\text { C_SANDEVI } \\
\text { CRS } \\
\text { MANESES } \\
\text { P_REUNIDOS } \\
\text { PC_CRIADO } \\
\text { TEBYCON } \\
\text { TECPROGESA } \\
\text { TEIDE } \\
\text { VALSAN }\end{array}$ & & $\begin{array}{l}\text { Endeudamiento alto } \\
\text { Neto sobre pasivo bajo } \\
\text { Liquidez inmediata y ratio de } \\
\text { circulante bajos } \\
\text { Rotación de activos, sobre todo } \\
\text { en } 2002 \text { y } 2003 \text { alta }\end{array}$ \\
\hline $\begin{array}{l}\text { Factor } 1+ \\
\text { Factor } 2 \text { - }\end{array}$ & $\begin{array}{l}\text { HORMISORIA } \\
\text { C_BELTRAN } \\
\text { CO_VALBUENA }\end{array}$ & $\begin{array}{l}\text { O_HERGON } \\
\text { A_NUMANCIA } \\
\text { PA_CASTILLA }\end{array}$ & & $\begin{array}{l}\text { Neto sobre pasivo alto } \\
\text { Liquidez inmediata alta } \\
\text { Endeudamiento bajo, pero } \\
\text { endeudamiento a corto plazo } \\
\text { alto } \\
\text { Gastos financieros sobre ventas } \\
\text { bajos } \\
\text { Prueba ácido } 2002 \text { y } 2003 \text { alta }\end{array}$ \\
\hline $\begin{array}{l}\text { Factor } 1+ \\
\text { Factor } 3+\end{array}$ & $\begin{array}{l}\text { UNI-ARQUITEC } \\
\text { C_BELTRAN } \\
\text { CO_VALBUENA }\end{array}$ & & & $\begin{array}{l}\text { Además de lo indicado, } \\
\text { Márgenes } 2002 \text { y } 2004 \text { bajos }\end{array}$ \\
\hline $\begin{array}{l}\text { Factor } 1+ \\
\text { Factor } 3 \text { - }\end{array}$ & $\begin{array}{l}\text { AVVIARE } \\
\text { C_GARMON } \\
\text { P_PAL }\end{array}$ & & & $\begin{array}{l}\text { Además de lo indicado, } \\
\text { Márgenes } 2002 \text { y } 2004 \text { altos }\end{array}$ \\
\hline
\end{tabular}

- Presentamos a continuación la imagen de los individuos compromiso con el TUCKALS3, con el fin de compararla con la imagen anterior. 
Figura 11: Imagen individuos-compromiso con TUCKALS3: plano 1-2 y plano 1-3, respectivamente

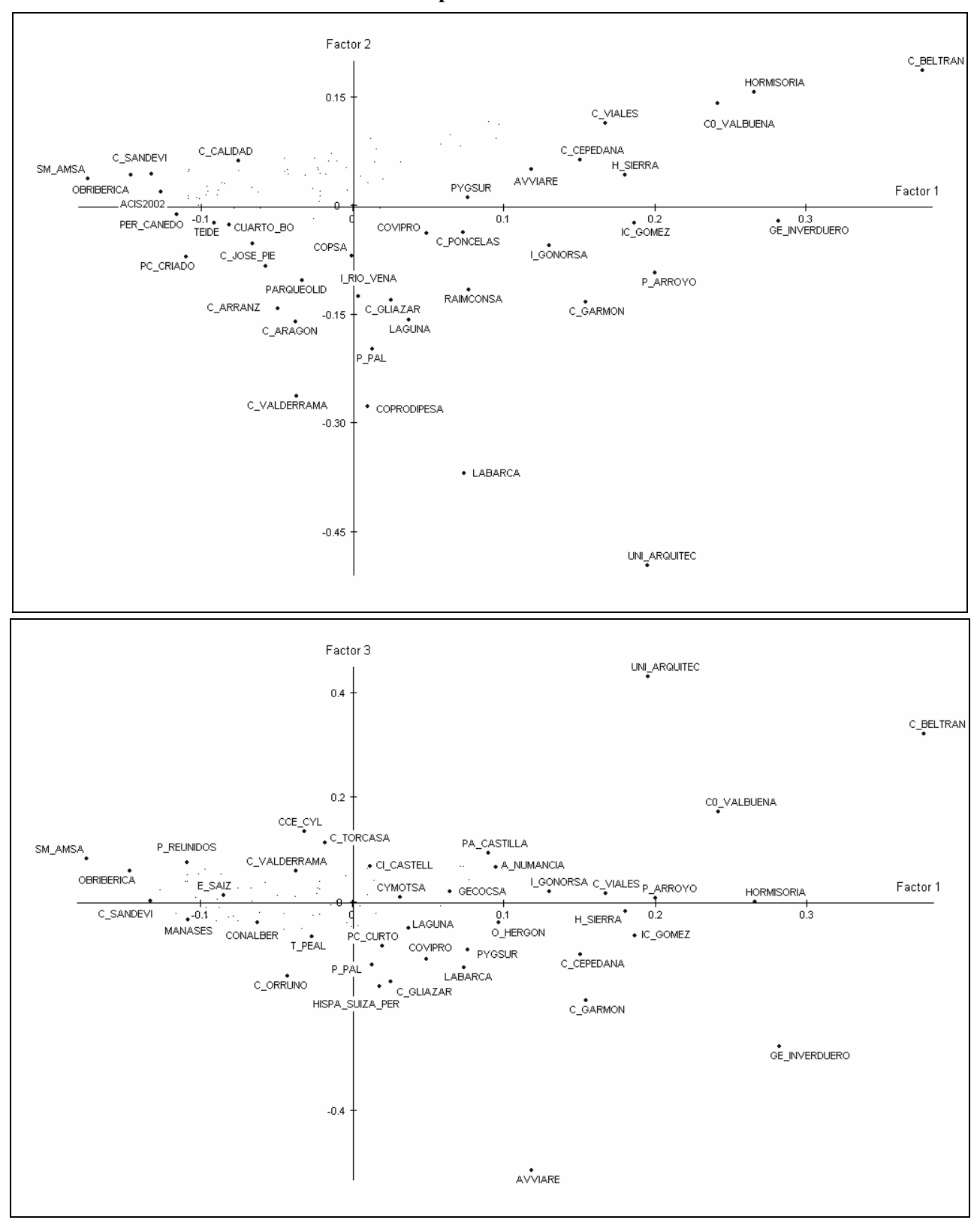

Como puede comprobarse, las posiciones en el plano de bastantes individuos coinciden con las descritas para la imagen de la figura 10 (presentadas en la tabla 6), aunque interpretadas en términos 
de distancias y proximidades. Sin embargo, en este caso no es posible relacionar individuos con variables. Lo mismo ocurre con la imagen obtenida con el método PARAFAC/CANDECOMP, por lo que se ha omitido dicha representación.

\subsubsection{Individuos: Trayectorias}

- Los métodos ${ }^{2}$ que permiten obtener la imagen de todos los individuos-parciales, y por tanto obtener trayectorias, son el AFM y el Statis dual. En las figuras 12 y 13 sólo se presentan los individuos de trayectoria más larga en el plano 1-2 (el plano 1-3 no aporta nada nuevo, por lo que no se presenta), así como la trayectoria de alguno de los individuos-compromiso que están situados en los extremos de los planos.

Figura 12: Trayectorias de los individuos en el AFM: plano 1-2

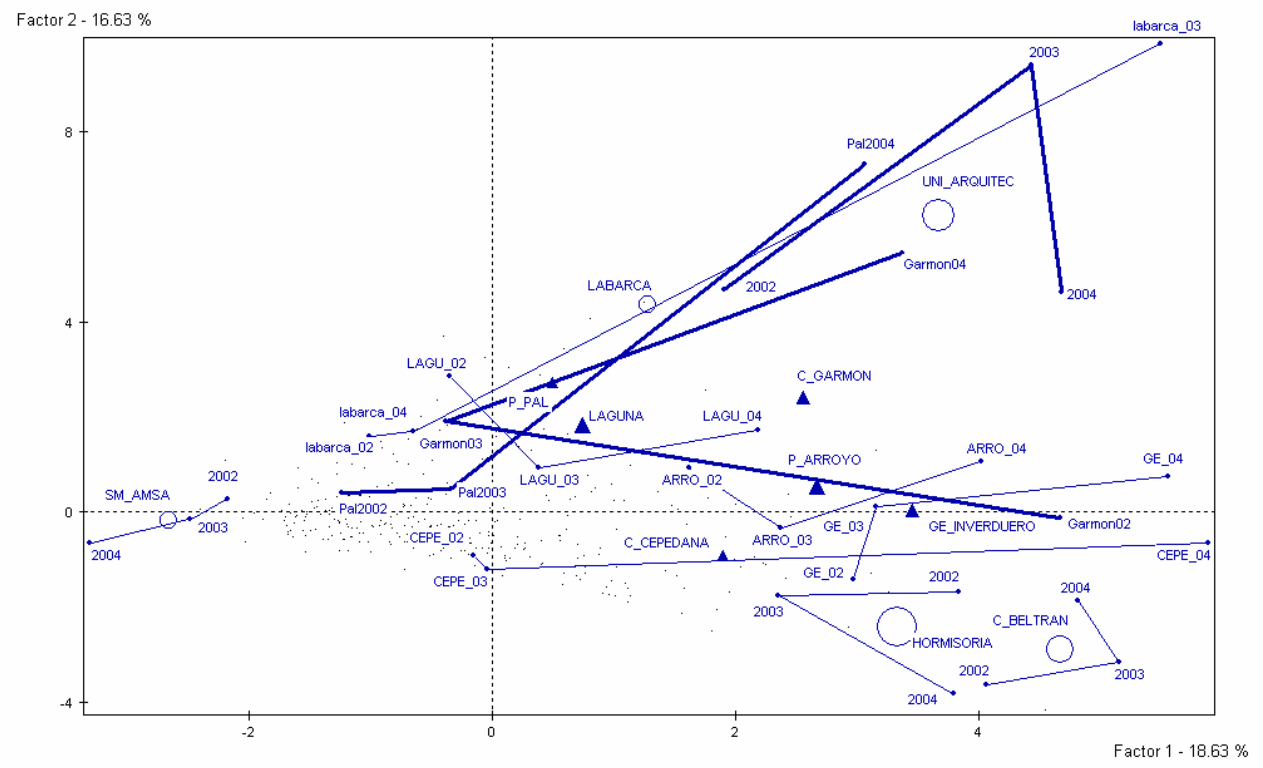

2 Como se ha comentado previamente, el trazado de trayectorias con la versión Statis resulta controvertido, motivo por él que no es considerado en este trabajo. 
Figura 13: Trayectorias de los individuos en el Statis dual: plano 1-2

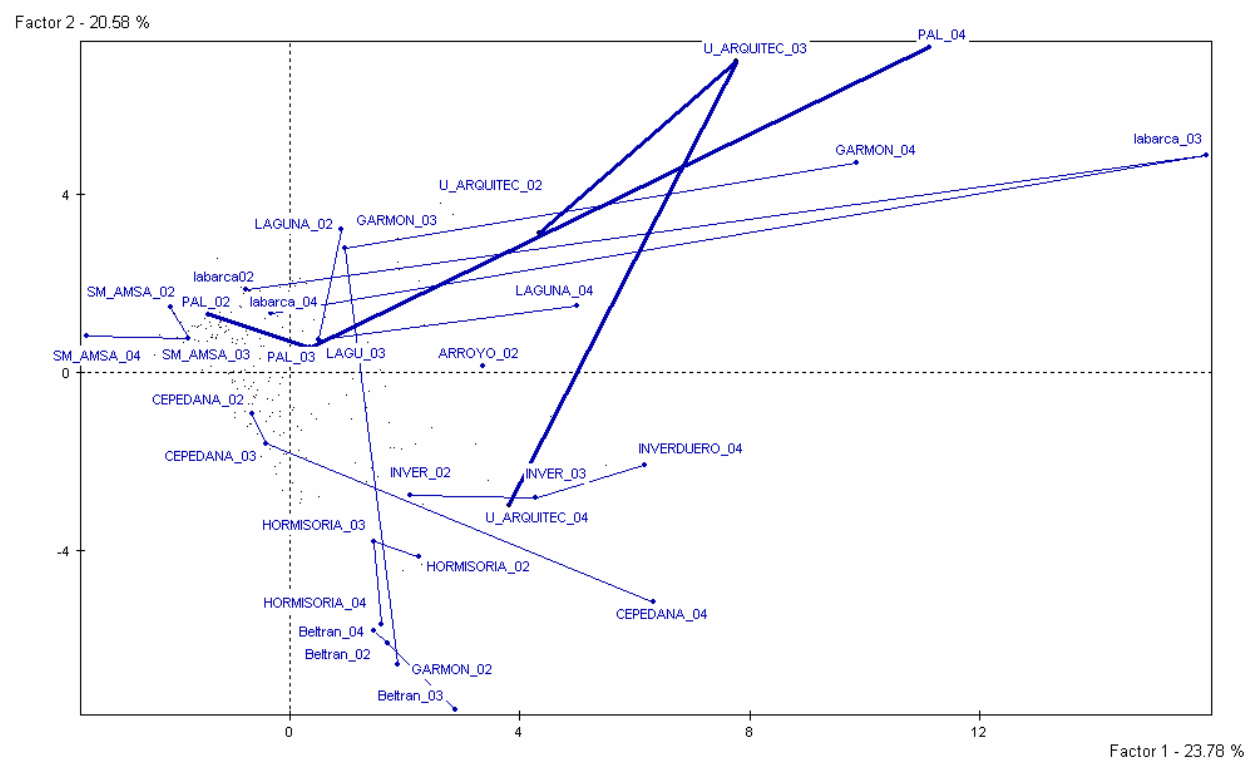

Como puede comprobarse, ambas figuras son similares. A diferencia del Statis dual, el AFM permite representar en el plano las posiciones compromiso de los individuos, lo que facilita la interpretación de las trayectorias. En cuanto a su trazado, las diferencias entre ambos métodos se deben al diferente significado de los factores que configuran el plano: en el caso del AFM las trayectorias son más largas que las del Statis dual respecto al factor 1 , y más cortas respecto al factor 2 . Sin embargo, se obtienen conclusiones similares ya que el factor 1 del AFM y el factor 2 del Statis dual se identificaba ambos como ESTRUCTURA DE PASIVO. Se han destacado en ambas figuras las trayectorias de algunos individuos para que la comparación de las gráficas resulte más sencilla. También se han modificado sensiblemente algunas etiquetas identificativas para que los planos obtenidos sean más legibles.

\section{Sobre las empresas analizadas en el periodo}

Como era previsible, las constructoras que describen las trayectorias más largas son aquellas cuyo individuo-compromiso estaba en un lugar destacado del plano, pero con baja calidad de representación (según tabla 6). En la imagen del AFM, destacan LABARCA (respecto a los factores 1 y 2), C_GARMON (respecto al factor 1), C_CEPEDANA (respecto al 
factor 1), P_ARROYO (respecto al factor 1), LAGUNA (respecto al factor 1) y P_PAL (respecto al factor 2). En la imagen del Statis dual, destacan las trayectorias de estos mismos individuos, pero respecto al factor contrario.

También se han dibujado las trayectorias de UNI_ARQUITEC (respecto al factor 2), HORMISORIA (respecto a los factores 1 y 2), GE_INVERDUERO (respecto al factor 1), SM_AMSA (respecto al factor 1) y C_BELTRAN (respecto al factor 2), comprobando que para ambas imágenes describen trayectorias más cortas, ya que se trata de individuos destacados y bien representados en el plano. Además, las trayectorias cortas no aportan demasiado y es suficiente con la interpretación del individuo-compromiso.

Por otro lado, para casi todos los individuos, tanto para AFM como para Statis dual, el individuo-parcial relativo al año 2004 se sitúa más a la derecha del lado positivo del factor 1: para el AFM, esta posición es lógica, dada la correlación de ese lado del factor con las variables de actividad para el 2004, tal y como refleja la imagen de la figura 4. Así, las empresas C_CEPEDANA (Construcciones Cepedana, S.L.), LAGUNA (Laguna 2000, S.L.), P_PAL (Promociones PAL S.A.), P_ARROYO (Promociones Arroyo Fuensaldaña, S.L.), GE_INVERDUERO (Grupo empresarial INVERDUERO, S.L), LAGUNA (Laguna 2000, S.L.), UNI-ARQUITEC (Urbanismo, naves industriales y arquitectura, S.L.) presentan una mayor actividad en el 2004 respecto a los demás, pero seguramente también mayor importancia del neto sobre el pasivo y de la liquidez, teniendo en cuenta el significado del factor. Con los resultados del Statis dual, no es posible llegar a tanto detalle ya que el significado de los factores no se especifica por años, interpretando las trayectorias solamente en función del significado global de las variables que los definen.

Por otra parte, los individuos-parciales del 2003 imprimen un cambio brusco en algunas de las trayectorias, lo que indica un cambio de comportamiento en ese año respecto al 2002 ó respecto al 2004. Ese cambio ya se reflejaba la figura 4 de este trabajo, comprobando ahora cuáles son los individuos responsables del mismo.

\section{CONCLUSIONES}

Con el trabajo realizado se ha conseguido alcanzar el objetivo planteado: probar que las diferencias teóricas entre las técnicas para tablas three-way data array, se traducen en resultados empíricos similares, y sin duda, complementarios. 
Con el fin de ordenar las conclusiones más concretas del trabajo, vamos a distinguir entre conclusiones de tipo metodológico (relativas a los métodos aplicados) y conclusiones de tipo económico (relativas a la información analizada).

- Desde el punto de vista metodológico, para analizar tablas de tres entradas con los mismos individuos y variables en cada ocasión, la elección del método depende del objetivo del estudio en cuanto al nivel de desglose de los resultados que se desee obtener.

Los métodos PCA-SUP y TUCKALS2, tal y como se ha indicado, no son los más adecuados dado que dejan de lado un aspecto importante de la información: el tercer modo. Además, en muchos casos es ese modo el tiempo, y resulta de sumo interés en la mayoría de los estudios.

En cuanto a los métodos $\mathrm{ICl}$, el Statis (ambas versiones) y el AFM, son métodos que se prefieren a los métodos de componentes cuando lo que se busca es analizar detalladamente las relaciones entre tablas, variables e individuos. Como hemos comprobado, ambos métodos extraen las características generales de la información analizada, llegando a las conclusiones muy similares. Por tanto, si lo que interesa es una visión general de la estructura común de las tablas, podemos aplicar tanto AFM como Statis (la versión correspondiente según cuál sea el elemento común). El Statis permite una mejor comparación global de las tablas a través de una representación gráfica que es óptima. Sin embargo, el AFM parece el método más apropiado cuando lo que se busca es analizar, también la estructura particular de las tablas de datos, ya que se obtienen resultados mucho más desglosados.

Respecto a los métodos de componentes, los métodos TUCKALS3 y PARAFAC/CANDECOMP extraen resultados satisfactorios de cada modo por separado, pero difíciles de interpretar cuando lo que se busca es relacionar individuos y variables. Además, ninguno de ellos permite estudiar los individuos y las variables parciales, sino sólo las posiciones medias. Por otro lado, los resultados que se extraen quedan limitados a las puntuaciones factoriales, sin otras ayudas a la interpretación. Son los métodos más apropiados cuando lo que interesa es el modelo global subyacente, pero no para describir detalladamente los datos analizados.

- En cuanto a las conclusiones económicas, matizar que deben completarse con trabajos posteriores ya que el objetivo primordial en este caso no es describir exhaustivamente las empresas constructoras estudiadas. Para obtener una visión completa habrá que interpretar todos 
los resultados presentados, además de analizar otros planos factoriales para individuos, variables y trayectorias. Para describir adecuadamente los individuos serán necesarias otras técnicas, como el Análisis Cluster, puesto que al ser muy numerosos las representaciones factoriales resultan poco legibles.

Por tanto, nos limitamos a las tendencias generales que se han destacado en el periodo 2002 y 2004, marcadas por los factores de mayor inercia, y que se concretan en las siguientes:

- El cambio más significativo se ha producido en el año 2003, que a todas luces es el que más difiere. Este cambio parece estar basado en una menor actividad o gestión de las empresas, como señalan los ratios relativos al margen bruto, comercial y beneficios sobre ventas.

- Se detecta cierta estabilidad en el periodo 2002-04 en la estructura del pasivo (neto sobre el pasivo, endeudamiento a corto y largo plazo), en la liquidez y en el peso de los gastos financieros sobre las ventas.

- También se pone de manifiesto una clara vinculación entre la mayor capacidad de la empresa para hacer frente a los pagos del siguiente ciclo de explotación y la mayor disponibilidad de tesorería.

- En cuanto a los individuos, los cambios más significativos en el periodo respecto a la tendencia general señalada los presentan Labarca-2 y asociados S.L., Construcciones Cepedana, S.L., Laguna 2000, S.L., Promociones PAL S.A., Promociones Arroyo Fuensaldaña, S.L., Grupo empresarial INVERDUERO, S.L, Laguna 2000, S.L. y Urbanismo, naves industriales y arquitectura, S.L.

Por tanto, la técnica más completa es el Análisis Factorial Múltiple y la aplicación de las demás enriquece los resultados obtenidos. Sin embargo, en la mayoría de los estudios es suficiente con la aplicación de la que resulte más apropiada para alcanzar los objetivos planteados.

\section{BIBLIOGRAFÍA}

Abascal Fernández, E.; LandaluCe Calvo, M.I. (2002) "Análisis Factorial Múltiple como técnica de estudio de la estabilidad de los resultados de un Análisis Componentes Principales", Questiió, 26, 1-2, pp. 109-122. 
BOVE, G.; DI CIACCIO, A. (1994) "A user-oriented overview of multiway methods and software", Computational Statistics and data Analysis,18, pp. 15-37.

CASIN, P. (2001) "A generalization of principal component analysis to $\mathrm{K}$ sets of variables", Computational Statistics and Data Analysis, 33, pp. 417-428.

Claver Cortés, E.; Molina Azorín, J.F.; Quer Ramón, D. (2002) "Grupos estratégicos, resultados empresariales y niveles de riesgo. Análisis empírico del sector de la construcción", Economía Industrial, 345, pp. 147-158.

ESCOFIER, B.; PAGĖS, J. (1992) Análisis factoriales simples y múltiples. Objetivos, métodos e interpretación. Bilbao: Ed. Servicio editorial de la Universidad del País Vasco.

EsCoufier, Y. (1976) "Opérateur associé à un tableau de données", Annales de l'INSEE, 22-23, pp. 165-178.

FUNDACIÓN UNIVERSIDAD CARLOS III (2004) "Análisis de la pequeña y mediana empresa del sector de construcción de la comunidad de Madrid", Papeles de trabajo.

GLAÇON, F. (1981) "Analyse conjointe de plusieurs matrices de données. Comparasion de différentes methods", Thèse de troisiéme cycle, Université de Grenoble.

HARSHMAN, R.A.; LUNDY, M.E. (1994) "PARAFAC: Parallel Factor Analysis", Computational Statistics and Data Analysis, 18, pp. 39-72.

KIERS, H.A.L. (1988) "Comparison of anglo-saxon and french three-mode methods", Statistique et Analyse des Données 13, 3, pp. 14-32.

- (1991) "Hierarchical relations among three-way methods", Psychometrika, 56, pp. 449-470.

KROONENBER, P.M. (1994) "The TUCKALS line. A suite of programs for threeway data Analysis", Computational Statistics and Data Analysis, 18, pp. 73-96.

LAVIT, Ch. (1988) Analyse conjointe de tableaux quantitatifs. Paris: Ed. Masson.

L'Hermier des Plantes, H. (1976) "Structuration des Tableaux à Trois Indices de la Statistique", Thése de 3ème cycle, Université des Sciences et Techniques du Languedoc.

PAGES, J. (1996) "Eléments de comparaison entre l'analyse factorielle multiple et la méthode STATIS", Revue de statistique Appliquée, XLIV(4), pp. 81-95. 
RIDRUEJO, Z.J. (2006) "Síntesis de la coyuntura económica en Castilla y León, 1 trimestre de 2006", Accesible (octubre 2006) en: www.psoecyl. org/imagenes/documentos/economia/06-06-28_iv_coyuntura.pdf.

VAllejo PAsCuAL, M.E. (2000) "Desarrollo de la metodología Statis. Aplicación al estudio del sector asegurador español", Tesis doctoral, Universidad de León. 


\section{ANEXO}

Tabla 1: Empresas constructoras analizadas

\begin{tabular}{|c|c|}
\hline CONSTRUCTORA & ETIQUETA \\
\hline ACIS $2002 \mathrm{SL}$ & ACIS2002 \\
\hline AGLOMERADOS NUMANCIA SL & A_NUMANCIA \\
\hline AGRUPACION RAMIREZ SL & A RAMIREZ \\
\hline ALGESA SA & ALGESA \\
\hline ALTAMIRA SL & ALTAMIRA \\
\hline ALVAC SA & ALVAC \\
\hline ARCEBANSA SA & ARCEBANSA \\
\hline AVVIARE HOLDING XXI SL & AVVIARE \\
\hline BEGAR CONSTRUCCIONES Y CONTRATAS SA & BEGAR \\
\hline BENITO OLALLA CONSTRUCCIONES SA & BENITO OLA \\
\hline BROQUEL SL & BROQUEL \\
\hline COFRENSA SL & CONFRENSA \\
\hline CONALBER SA & CONALBER \\
\hline CONSERVACION DE VIALES SA & C_VIALES \\
\hline CONSERVACION E INFRAESTRUCTURAS CASTELLANAS SA & CI CASTELL \\
\hline CONSTRUCCION INTEGRAL DE FIRMES CPA SA & CI FIRMES \\
\hline CONSTRUCCIONES ARAGON IZQUIERDO SL & C_ARAGON \\
\hline CONSTRUCCIONES ARRANZ ACINAS SA & C_ARRANZ \\
\hline CONSTRUCCIONES BELTRAN MONUX SA & C BELTRAN \\
\hline CONSTRUCCIONES CARRIEGOS SA & C CARRIEGOS \\
\hline CONSTRUCCIONES CIVILES EOLICAS DE CASTILLA Y LEON SA & CCE_CYL \\
\hline CONSTRUCCIONES DEL DURATON SA & C DURATON \\
\hline CONSTRUCCIONES GARMON Y LLAMAS SL & C GARMON \\
\hline CONSTRUCCIONES GLIAZAR SL & C_GLIAZAR \\
\hline CONSTRUCCIONES HERMANOS SASTRE SA & C_HER SASTRE \\
\hline CONSTRUCCIONES JOSE PIEDRA SA & C_JOSE_PIE \\
\hline CONSTRUCCIONES ORRUNO SA & C_ORRUNO \\
\hline CONSTRUCCIONES PONCELAS SA & C_PONCELAS \\
\hline CONSTRUCCIONES RAMON GARCIA SL & C RAMON GAR \\
\hline CONSTRUCCIONES SANDEVI SL & C_SANDEVI \\
\hline CONSTRUCCIONES TORCA SA & C TORCASA \\
\hline CONSTRUCCIONES VALDERRAMA SA & C VALDERRAMA \\
\hline CONSTRUCCIONES Y MONTAJES NOROESTE SA & CM_NOROESTE \\
\hline CONSTRUCCIONES Y OBRAS LLORENTE SA & C_LLORENTE \\
\hline CONSTRUCCIONES Y OBRAS VALBUENA SA & $\mathrm{C} 0$ VALBUENA \\
\hline CONSTRUCTORA CEPEDANA SL & C_CEPEDANA \\
\hline CONSTRUCTORA PEACHE SA & C_PEACHE \\
\hline CONTRATAS Y MAQUINARIA SA & C MAQU \\
\hline CONTRATAS Y OBRAS SAN GREGORIO SA & CO SAN GREGO \\
\hline COPRODIPESA SA & COPRODIPESA \\
\hline COPSA EMPRESA CONSTRUCTORA SA & COPSA \\
\hline COVIPRO SL & COVIPRO \\
\hline CRIVASA SA & CRIVASA \\
\hline CRS SA & CRS \\
\hline CUARTO BOCEL SL & CUARTO_BO \\
\hline CYMOT SA & CYMOTSA \\
\hline DESARROLLOS INTEGRALES DE CASTILLA Y LEON SA & C_CALIDAD \\
\hline ESTEBAN FERNANDEZ E HIJOS SA & ESTEBAN_FER \\
\hline EXCAVACIONES SAIZ SA & E SAIZ \\
\hline GASINDUR SL & GASINDUR \\
\hline GECOCSA GENERAL DE CONSTRUCCIONES CIVILES SA & GECOCSA \\
\hline
\end{tabular}




\begin{tabular}{|c|c|}
\hline CONSTRUCTORA & ETIQUETA \\
\hline GRUPO EMPRESARIAL INVERDUERO SL & GE_INVERDUERO \\
\hline HERMANOS TABARA SL & H TABARA \\
\hline HERMERIEL SA & HERMERIEL \\
\hline HISPANA SUIZA DE PERFILADOS SA & HISPA_SUIZA_PER \\
\hline HORMIGONES SIERRA SL & H_SIERRA \\
\hline HORMISORIA SL & HORMISORIA \\
\hline INMOBILIARIA CONSTRUCTORA GOMEZ Y GOMEZ SA & IC GOMEZ \\
\hline INMOBILIARIA GONORSA SA & I_GONORSA \\
\hline INMOBILIARIA RIO VENA SA & I_RIO_VENA \\
\hline INSTALACIONES SOLINOX ARANDINAS SL & I SOLINOX \\
\hline LABARCA-2 Y ASOCIADOS SL & LABARCA \\
\hline LAGUNA $2000 \mathrm{SL}$ & LAGUNA \\
\hline MANANES INSTALACIONES PROYECTOS Y SERVICIOS SA & MANASES \\
\hline MARIANO RICO SL & MARIANO \\
\hline OBRAS HERGON SA & O_HERGON \\
\hline OBRAS Y ESTRUCTURAS RAM SL & OE_RAM \\
\hline OBRIBERICA SL & OBRIBERICA \\
\hline PARQUEOLID PROMOCIONES SA & PARQUEOLID \\
\hline PAVIMENTOS ASFALTICOS DE CASTILLA SA & PA_CASTILLA \\
\hline PAVIMENTOS ASFALTICOS SALAMANCA SL & PA_SALAMAN \\
\hline PEREZ CANEDO SA & PER_CANEDO \\
\hline PRIA SA & PRIASA \\
\hline PROMOCIONES ARROYO FUENSALDANA SL & P_ARROYO \\
\hline PROMOCIONES PAL SA & P.PAL \\
\hline PROMOCIONES Y CONTRATAS CURTO SL & PC_CURTO \\
\hline PROMOCIONES Y REFORMAS CRIADO SL & PC_CRIADO \\
\hline PROYECTOS Y CONSTRUCCIONES REUNIDOS SA & P REUNIDOS \\
\hline PYGSUR PROMOCION Y GESTION DE SERVICIOS URBANOS SA & PYGSUR \\
\hline RAIMCONSA PROMOCIONES SA & RAIMCONSA \\
\hline SALMANTINA DE SEGURIDAD VIAL SA & SALMANTINA_SEG \\
\hline SISTEMAS Y MONTAJES AMSA SL & SM_AMSA \\
\hline SYH CONSTRUCCION SERVICIOS Y MEDIO AMBIENTE SA & SYHSA \\
\hline TEBYCON SA & TEBYCON \\
\hline TECNICOS UNIDOS PARA LA CONSTRUCCION SA & T_U_CONSTRU \\
\hline TECPROGESA SA & TECPROGESA \\
\hline TEIDE $90 \mathrm{SL}$ & TEIDE \\
\hline TRANSPORTES PEAL SA & T_PEAL \\
\hline URBANISMO NAVES INDUSTRIALES Y ARQUITECTURA SL & UNI_ARQUITEC \\
\hline VALSAN CONSTRUCCIONES Y CONTRATAS SL & VALSAN \\
\hline VENTILACION ESTRUCTURA Y MONTAJES METALICOS SL & VE_MONTA_META \\
\hline VOLCONSA CONSTRUCCION Y DESARROLLO DE SERVICIOS SA & VOLCONSA \\
\hline ZARZUELA SA EMPRESA CONSTRUCTORA & ZARZUELA \\
\hline
\end{tabular}


Tabla 2: Coordenadas y contribuciones absolutas del Análisis Factorial Múltiple

\begin{tabular}{|l||c|c|c||c|c|c|}
\hline \multicolumn{7}{|c|}{ ANO 2004 } \\
\hline \multicolumn{1}{|c||}{ Variables } & \multicolumn{3}{c|}{ Coordenadas } & \multicolumn{2}{c|}{ Contribuciones absolutas } \\
\cline { 2 - 7 } & Eje 1 & Eje 2 & Eje 3 & Eje 1 & Eje 2 & Eje 3 \\
\hline \hline ROA_2004 & 0,5153 & 0,0620 & $-0,3763$ & 2,6408 & 0,0429 & 2,8682 \\
ROE_2004 & 0,1779 & 0,4894 & 0,0407 & 0,3149 & 2,6687 & 0,0336 \\
MBR_2004 & 0,5713 & 0,1008 & $-0,1839$ & 3,2458 & 0,1132 & 0,6850 \\
MCO_2004 & 0,6113 & 0,0250 & $-0,1779$ & 3,7161 & 0,0070 & 0,6410 \\
RBV_2004 & 0,6183 & 0,0334 & $-0,1395$ & 3,8022 & 0,0125 & 0,3940 \\
RCI_2004 & 0,5924 & 0,5608 & 0,2443 & 3,4901 & 3,5043 & 1,2090 \\
PAC_2004 & 0,5139 & $-0,2031$ & 0,0891 & 2,6262 & 0,4598 & 0,1610 \\
LIQ_2004 & 0,6083 & 0,1357 & $-0,0487$ & 3,6801 & 0,2053 & 0,0479 \\
CCV_2004 & 0,2670 & 0,3331 & 0,3189 & 0,7088 & 1,2362 & 2,0603 \\
RCA_2004 & $-0,1788$ & 0,2515 & 0,2885 & 0,3179 & 0,7046 & 1,6863 \\
CCF_2004 & 0,2634 & $-0,2045$ & $-0,0836$ & 0,6898 & 0,4658 & 0,1416 \\
NPA_2004 & 0,7503 & $-0,4194$ & $-0,2067$ & 5,5983 & 1,9598 & 0,8650 \\
REP_2004 & $-0,7484$ & 0,4232 & 0,2511 & 5,5702 & 1,9961 & 1,2767 \\
RECP_2004 & $-0,3166$ & $-0,6659$ & $-0,1879$ & 0,9970 & 4,9410 & 0,7153 \\
PMD_2004 & $-0,1990$ & $-0,1554$ & 0,1267 & 0,3938 & 0,2690 & 0,3253 \\
RAC_2004 & $-0,2263$ & $-0,0436$ & $-0,2162$ & 0,5093 & 0,0212 & 0,9468 \\
GFV_2004 & $-0,1717$ & 0,5988 & 0,0304 & 0,2932 & 3,9954 & 0,0187 \\
DVE_2004 & 0,1850 & $-0,3509$ & 0,0375 & 0,3403 & 1,3723 & 0,0284 \\
\hline \hline GRUPO & & & & 38,9349 & 23,9746 & 14,1040 \\
\hline
\end{tabular}

\begin{tabular}{|c|c|c|c|c|c|c|}
\hline \multicolumn{7}{|c|}{ AÑNO 2003} \\
\hline \multirow[b]{2}{*}{ Variables } & \multicolumn{3}{|c|}{ Coordenadas } & \multicolumn{3}{|c|}{ Contribuciones absolutas } \\
\hline & Eje 1 & Eje 2 & Eje 3 & Eje 1 & Eje 2 & Eje 3 \\
\hline ROA_ 2003 & 0,5348 & $-0,3401$ & $-0,0120$ & 2,6282 & 1,1909 & 0,0027 \\
\hline ROE_ 2003 & $-0,1540$ & 0,2438 & 0,0186 & 0,2180 & 0,6117 & 0,0064 \\
\hline MBR_2003 & 0,3357 & 0,4844 & 0,1538 & 1,0357 & 2,4154 & 0,4425 \\
\hline MCO_2003 & 0,3328 & 0,2308 & 0,1247 & 1,0177 & 0,5483 & 0,2908 \\
\hline RBV_2003 & 0,3446 & 0,1924 & 0,0747 & 1,0913 & 0,3812 & 0,1043 \\
\hline RCI_2003 & 0,6357 & 0,4957 & 0,4335 & 3,7137 & 2,5299 & 3,5172 \\
\hline PAC̄_2003 & 0,3297 & $-0,5367$ & 0,2469 & 0,9986 & 2,9654 & 1,1407 \\
\hline LIQ_-2003 & 0,5592 & $-0,3522$ & 0,2192 & 2,8732 & 1,2767 & 0,8995 \\
\hline CCV_2003 & 0,3326 & 0,6868 & 0,3763 & 1,0168 & 4,8558 & 2,6502 \\
\hline RCA_2003 & 0,6357 & 0,4957 & 0,4335 & 3,7137 & 2,5299 & 3,5172 \\
\hline CCF_2003 & 0,2022 & $-0,2335$ & 0,0463 & 0,3758 & 0,5615 & 0,0401 \\
\hline NPA_2003 & 0,6833 & $-0,5910$ & 0,1045 & 4,2902 & 3,5964 & 0,2043 \\
\hline REP_2003 & $-0,5562$ & 0,4740 & $-0,0878$ & 2,8431 & 2,3133 & 0,1442 \\
\hline RECP_ 2003 & $-0,2950$ & $-0,6009$ & $-0,3578$ & 0,7998 & 3,7176 & 2,3958 \\
\hline PMD_2003 & 0,1861 & 0,5840 & 0,1292 & 0,3183 & 3,5109 & 0,3124 \\
\hline RAC_- 2003 & $-0,4476$ & $-0,4611$ & 0,0309 & 1,8410 & 2,1892 & 0,0178 \\
\hline GFV_2003 & 0,1668 & 0,7584 & 0,1328 & 0,2556 & 5,9214 & 0,3299 \\
\hline DVE_2003 & 0,3968 & 0,1812 & 0,1041 & 1,4468 & 0,3380 & 0,2028 \\
\hline GRUPO & & & & 30,4774 & 41,4537 & 16,2189 \\
\hline
\end{tabular}




\begin{tabular}{|l||r|r|r||c|c|c|}
\hline \multicolumn{7}{|c|}{ ANO 2002 } \\
\hline \multirow{2}{*}{ Variables } & \multicolumn{3}{c|}{ Coordenadas } & \multicolumn{2}{c|}{ Contribuciones absolutas } \\
\cline { 2 - 6 } & Eje 1 & Eje 2 & Eje 3 & Eje 1 & Eje 2 & \multicolumn{1}{|c||}{ Eje 3 } \\
\hline \hline ROA_2002 & 0,3822 & $-0,3748$ & 0,0121 & 1,5600 & 1,6810 & 0,0032 \\
ROE_2002 & 0,2250 & 0,1990 & 0,1712 & 0,5409 & 0,4741 & 0,6379 \\
MBR_2002 & 0,3606 & 0,3358 & $-0,8000$ & 1,3889 & 1,3498 & 13,9197 \\
MCO_2002 & 0,3979 & $-0,1930$ & $-0,2675$ & 1,6908 & 0,4456 & 1,5566 \\
RBV_2002 & 0,3606 & 0,3358 & $-0,8000$ & 1,3889 & 1,3498 & 13,9197 \\
RCI_2002 & 0,6079 & 0,3478 & 0,2253 & 3,9471 & 1,4480 & 1,1044 \\
PAC_2002 & 0,3182 & $-0,5247$ & 0,2351 & 1,0816 & 3,2948 & 1,2022 \\
LIQ_2002 & 0,5479 & $-0,3427$ & 0,2380 & 3,2070 & 1,4059 & 1,2324 \\
CCV_2002 & 0,2231 & 0,4952 & $-0,4042$ & 0,5318 & 2,9347 & 3,5547 \\
RCA_2002 & $-0,1439$ & 0,3184 & 0,1127 & 0,2211 & 1,2130 & 0,2763 \\
CCF_2002 & 0,3412 & $-0,3864$ & 0,1180 & 1,2433 & 1,7872 & 0,3027 \\
NPA_2002 & 0,6619 & $-0,5848$ & 0,1019 & 4,6801 & 4,0933 & 0,2260 \\
REP_2002 & $-0,6608$ & 0,5858 & $-0,0986$ & 4,6639 & 4,1064 & 0,2114 \\
RECP_2002 & $-0,2950$ & $-0,6009$ & $-0,3578$ & 0,9297 & 4,3214 & 2,7849 \\
PMD_2002 & 0,0850 & 0,2977 & $-0,6129$ & 0,0772 & 1,0609 & 8,1713 \\
RAC_2002 & $-0,4242$ & $-0,3230$ & 0,1176 & 1,9220 & 1,2489 & 0,3010 \\
GFV_2002 & 0,2444 & 0,4265 & $-0,7575$ & 0,6381 & 2,1770 & 12,4803 \\
DVE_2002 & 0,2863 & 0,1226 & $-0,5985$ & 0,8753 & 0,1799 & 7,7927 \\
\hline \hline GRUPO & & & & 30,5878 & 34,5718 & 69,6771 \\
\hline
\end{tabular}

